\title{
Islamic Republic of Mauritania: Statistical Appendix
}

This Statistical Appendix for the Islamic Republic of Mauritania was prepared by a staff team of the International Monetary Fund as background documentation for the periodic consultation with the member country. It is based on the information available at the time it was completed on June 7 , 2006. The views expressed in this document are those of the staff team and do not necessarily reflect the views of the government of the Islamic Republic of Mauritania or the Executive Board of the IMF.

The policy of publication of staff reports and other documents by the IMF allows for the deletion of market-sensitive information.

To assist the IMF in evaluating the publication policy, reader comments are invited and may be sent by e-mail to publicationpolicy@imf.org.

Copies of this report are available to the public from

International Monetary Fund $\bullet$ Publication Services

700 19th Street, N.W. • Washington, D.C. 20431

Telephone: (202) $6237430 \bullet$ Telefax: (202) 6237201

E-mail: publications@imf.org • Internet: http://www.imf.org

Price: $\$ 15.00$ a copy

\section{International Monetary Fund Washington, D.C.}





\title{
INTERNATIONAL MONETARY FUND
}

\section{ISLAMIC REPUBLIC OF MAURITANIA}

\begin{abstract}
Statistical Appendix
Prepared by a staff team consisting of Jean Le Dem, Jaroslaw Wieczorek, Boileau Loko, Roua Hijazi (All MCD), and Andre Meier (PDR)
\end{abstract}

Approved by Middle East and Central Asia Department

June 7, 2006

Contents

Page

1. Gross Domestic Product by Activity at Current Prices, 1992-2005 .............................3

2. Gross Domestic Product by Activity at 1998 Prices, 1992-2005 .................................. 4

3. Growth of Output by Activity at 1998 Prices, 1993-2005 .........................................5

4. Area Cultivated and Production of Selected Crops, 1999/00-2005/06 .........................6

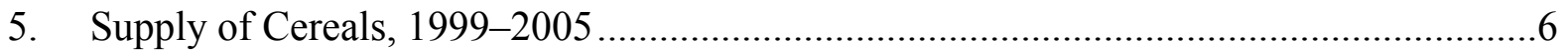

6. Estimated Size of Livestock Herds, Controlled Slaughtering,

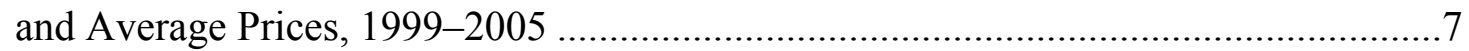

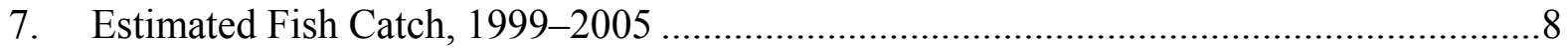

8. Iron Ore-Production, Exports, and Stocks, 1999-2005 ........................................

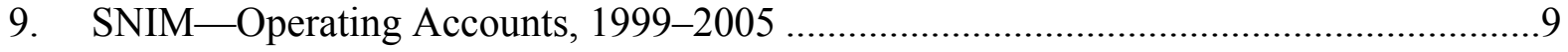

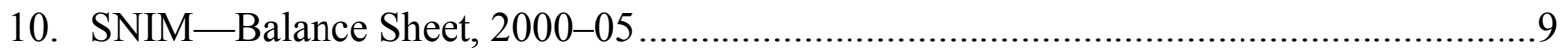

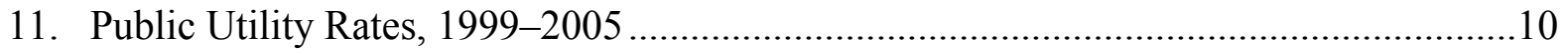

12. Consumption of Petroleum Products, 1999-2005 …................................................. 10

13. Unit Prices of Petroleum Products, 2001-06 ................................................................ 11

14. Consumer Price Index for Nouakchott, January 2004-April 2006..............................12

15. Evolution of Minimum Wages and Salaries, 1999-2005 ........................................... 13

16. Consolidated Government Operations, 1994-2005 ..................................................14

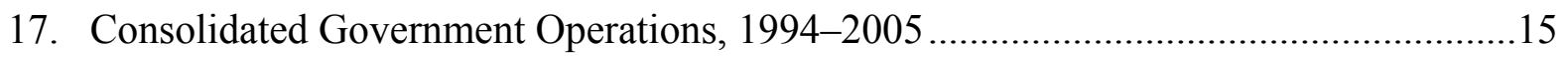

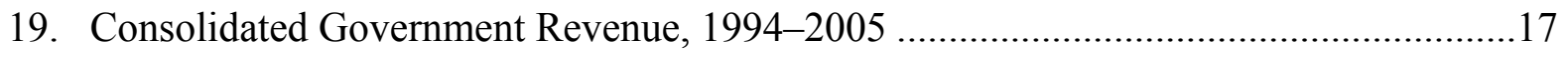

20. Deposit Money Banks, as of end-December 2005 ................................................. 18

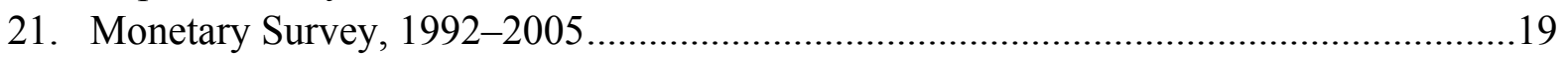

22. Assets and Liabilities of the Central Bank, 1992-2005 ............................................20

23. Assets and Liabilities of Commercial Banks, 1992-2005 ..........................................21

24. Foreign Assets and Liabilities of the Banking System, 1992-2005 .............................22

25. Selected Lending and Deposit Interest Rates, 1992-2005.......................................23 
26. Distribution of Bank Credit by Maturity and Economic Activity, 1992-2005 ..............24

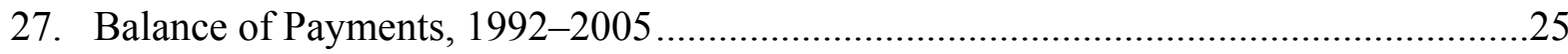

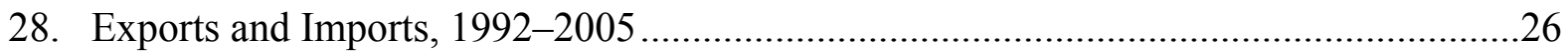

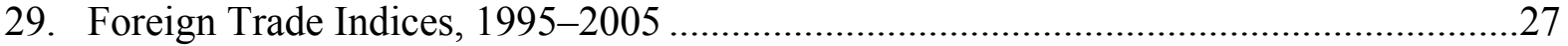

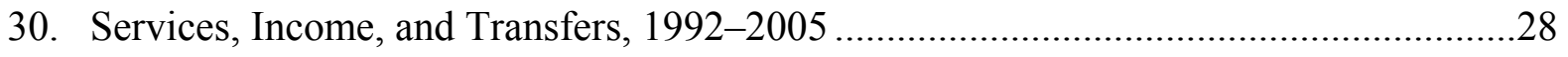

31. External Public/Publicly Guaranteed Debt Outstanding

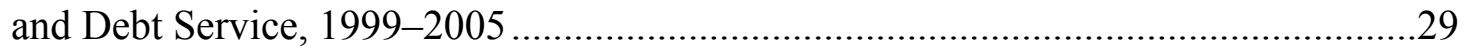

32. Medium- and Long-Term External Public/Publicly Guaranteed Debt, 19992005 ..........30

33. Average Terms of Contracted Public External Debt, 1999-2005 .................................31 


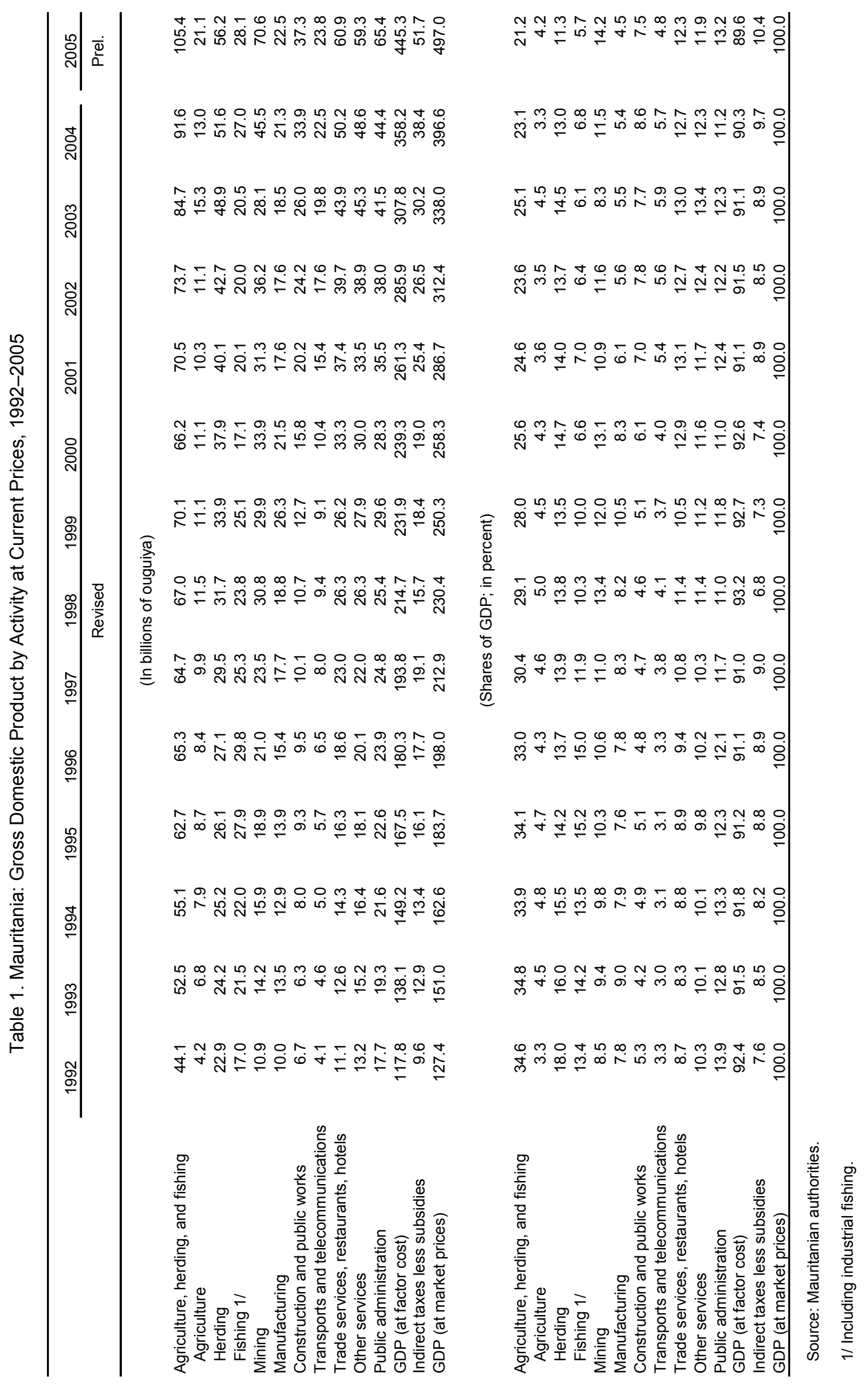




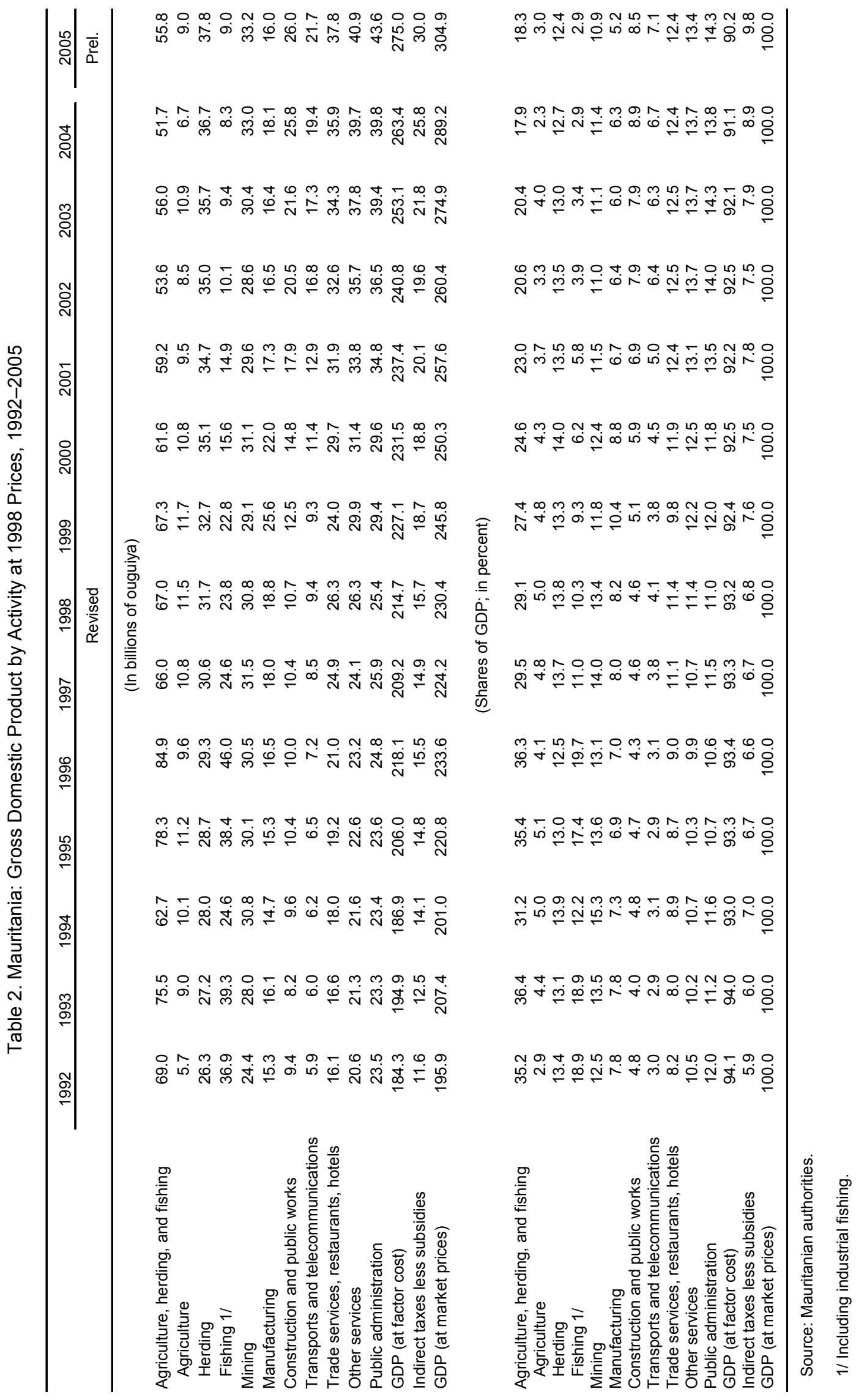




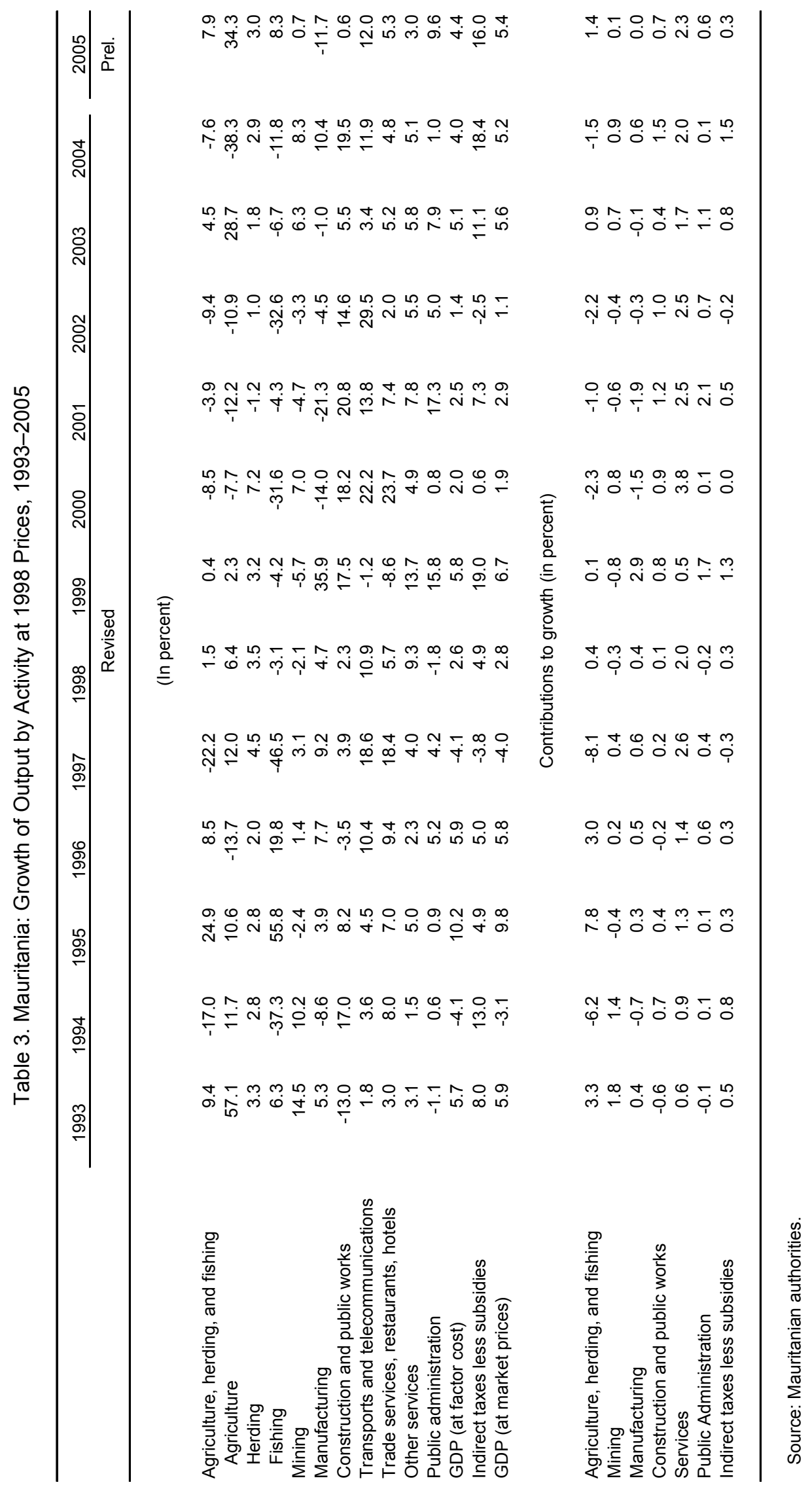


Table 4. Mauritania: Area Cultivated and Production of Selected Crops,1999/00-2005/06 1/

\begin{tabular}{|c|c|c|c|c|c|c|c|}
\hline & 1999/00 & $2000 / 01$ & $2001 / 02$ & $2002 / 03$ & $2003 / 04$ & $2004 / 05$ & $2005 / 06$ \\
\hline & \multicolumn{7}{|c|}{ (In thousands of hectares) } \\
\hline \multicolumn{8}{|l|}{ Area cultivated } \\
\hline Total cereals & 223.2 & 207.2 & 194.5 & 109.3 & 212.5 & 154.5 & 217.9 \\
\hline Millet and sorghum & 194.6 & 174.1 & 174.3 & 82.9 & 166.4 & 136.0 & 170.7 \\
\hline Paddy rice & 21.8 & 18.0 & 13.0 & 19.4 & 21.0 & 18.2 & 18.5 \\
\hline Maize, wheat, and barley & 6.8 & 15.1 & 7.2 & 7.0 & 25.1 & 0.3 & 28.7 \\
\hline Cowpeas, vegetables, and other & 46.6 & 37.7 & 39.5 & 25.5 & 28.5 & 30.0 & 26.0 \\
\hline \multirow[t]{2}{*}{ Dates } & $\ldots$ & $\ldots$ & $\ldots$ & $\ldots$ & $\ldots$ & $\ldots$ & $\ldots$ \\
\hline & \multicolumn{7}{|c|}{ (In thousands of metric tons) } \\
\hline \multicolumn{8}{|l|}{ Production } \\
\hline Total cereals & 192.8 & 178.5 & 124.8 & 115.8 & 181.2 & 114.8 & 192.8 \\
\hline Millet and sorghum & 101.2 & 88.7 & 57.7 & 26.9 & 83.8 & 20.2 & 97.8 \\
\hline Paddy rice & 86.5 & 76.2 & 58.8 & 85.3 & 78.9 & 85.5 & 72.0 \\
\hline Maize, wheat, and barley & 5.1 & 13.7 & 8.3 & 3.6 & 18.5 & 9.1 & 23.0 \\
\hline Cowpeas, vegetables, and other & 70.0 & 68.1 & 53.0 & 52.5 & 55.0 & 47.5 & 52.6 \\
\hline Dates & 20.0 & 18.0 & 22.0 & 22.0 & 22.0 & 20.0 & 8.0 \\
\hline
\end{tabular}

Source: Ministry of Rural Development and Environment (MDRE).

$1 /$ Crop season is from October through September.

Table 5. Mauritania: Supply of Cereals, 1999-2005

(In thousands of metric tons)

\begin{tabular}{|c|c|c|c|c|c|c|c|}
\hline & 1999 & 2000 & 2001 & 2002 & 2003 & 2004 & 2005 \\
\hline Production 1/ & 191.2 & 194.6 & 179.9 & 120.4 & 142.5 & 178.4 & 192.8 \\
\hline Imports & 209.7 & 269.8 & 247.7 & 259.1 & 355.3 & 179.8 & 423.1 \\
\hline Rice & 28.0 & 29.1 & 9.5 & 15.2 & 0.0 & 22.0 & 45.3 \\
\hline Flour & 181.7 & 240.6 & 238.2 & 243.9 & 355.3 & 156.4 & 370.7 \\
\hline Other & 0.0 & 0.0 & 0.0 & 0.0 & 0.0 & 1.4 & 7.1 \\
\hline Food aid & 27.7 & 20.9 & 22.7 & 34.3 & 74.1 & 46.6 & 49.0 \\
\hline Total supply & 428.7 & 485.3 & 450.3 & 413.8 & 571.9 & 404.8 & 664.9 \\
\hline Per capita supply (in kilograms) & 147.0 & 162.0 & 154.8 & 134.2 & 175.6 & 132.4 & 183.9 \\
\hline
\end{tabular}

Sources: Commission of Food Security (CSA); Ministry of Rural Development and Environment (MDRE); and Customs Directorate (DGD).

$1 /$ Including change in stocks. 
Table 6. Mauritania: Estimated Size of Livestock Herds, Controlled Slaughtering, and Average Prices, 1999-2005

\begin{tabular}{|c|c|c|c|c|c|c|c|}
\hline & 1999 & 2000 & 2001 & 2002 & 2003 & 2004 & 2005 \\
\hline & \multicolumn{7}{|c|}{ (In thousands of heads) } \\
\hline \multicolumn{8}{|l|}{ Livestock herds $1 /$} \\
\hline Cattle & 1,497 & 1,550 & 1,620 & 1,676 & 1,315 & 1,353 & 1,363 \\
\hline Sheep and goats & 12,558 & 13,384 & 13,775 & 14,045 & 14,329 & 14,974 & 15,798 \\
\hline Camels & 1,230 & 1,278 & 1,329 & 1,381 & 1,323 & 1,349 & 1,376 \\
\hline \multicolumn{8}{|c|}{ Controlled slaughtering } \\
\hline Cattle & 59 & 61 & 64 & 67 & 68 & 69 & 218 \\
\hline Sheep and goats & 158 & 167 & 171 & 181 & 187 & 193 & ... \\
\hline Camels & 49 & 50 & 52 & 53 & 55 & 57 & 95 \\
\hline & \multicolumn{7}{|c|}{ (In ouguiya per head) } \\
\hline \multicolumn{8}{|l|}{ Average Price } \\
\hline Cattle & 29,350 & 38,500 & 39,848 & 41,242 & 39,863 & 40,318 & 43,430 \\
\hline Sheep & 7,000 & 7,280 & 8,450 & 8,511 & 8,080 & 8,347 & 9,200 \\
\hline Goats & 6,578 & 6,841 & 7,125 & 7,129 & 7,032 & 7,095 & 8,500 \\
\hline Camels & 52,386 & 62,350 & 64,532 & 66,791 & 64,558 & 65,294 & 70,200 \\
\hline
\end{tabular}

Source: Ministry of Rural Development and Environment (MDRE).

$1 /$ Stock at year-end. 
Table 7. Mauritania: Estimated Fish Catch, 1999-2005 1/

(In thousands of metric tons)

\begin{tabular}{|c|c|c|c|c|c|c|c|}
\hline & 1999 & 2000 & 2001 & 2002 & 2003 & 2004 & 2005 \\
\hline Artisanal fishing & 14,527 & 19,456 & 22,139 & 26,131 & 23,899 & 30,770 & 35,386 \\
\hline Traditional & 7,586 & 11,364 & 12,933 & 16,660 & 15,237 & $\ldots$ & $\ldots$ \\
\hline Modern & 6,941 & 8,092 & 9,206 & 9,471 & 8,662 & $\cdots$ & $\ldots$ \\
\hline Industrial fishing & 490,211 & 525,469 & 620,146 & 646,512 & 615,174 & 792,078 & 660,081 \\
\hline Demersal (deep-sea) fish & 20,471 & 19,320 & 26,414 & 20,414 & 36,106 & 33,112 & 58,640 \\
\hline Pelagic (surface) fish & 419,880 & 458,093 & 544,837 & 602,565 & 532,714 & 727,811 & 601,441 \\
\hline Other & 49,860 & 48,056 & 48,895 & 23,533 & 46,354 & 31,155 & $\ldots$ \\
\hline Total & 504,738 & 544,925 & 642,285 & 672,643 & 639,073 & 822,848 & 695,466 \\
\hline
\end{tabular}

Sources: Fisheries Resources Research and Management Department (DEARH); and Customs Directorate (DGD).

1/ Including catch by the EU fleet.

Table 8. Mauritania: Iron Ore_Production, Exports, and Stocks, 1999-2005

\begin{tabular}{|c|c|c|c|c|c|c|c|}
\hline & 1999 & 2000 & 2001 & 2002 & 2003 & 2004 & 2005 \\
\hline & \multicolumn{7}{|c|}{ (In thousands of metric tons) } \\
\hline Production & 10,401 & 11,345 & 10,302 & 9,553 & 10,153 & 10,674 & 10,752 \\
\hline Exports & 11,042 & 11,069 & 10,093 & 10,460 & 9,627 & 11,003 & 10,638 \\
\hline Changes in stocks & -641 & 276 & 209 & -907 & 526 & -329 & 114 \\
\hline \multirow[t]{2}{*}{ Stocks (end of period) } & 1,369 & 1,645 & 1,854 & 947 & 1,473 & 1,144 & 1,258 \\
\hline & \multicolumn{7}{|c|}{ (Percentage changes) } \\
\hline Production & -8.5 & 9.1 & -9.2 & -7.3 & 6.3 & 5.1 & 0.7 \\
\hline Exports & -3.2 & 0.2 & -8.8 & 3.6 & -8.0 & 14.3 & -3.3 \\
\hline
\end{tabular}

Source: National Industrial and Mining Company (SNIM). 
Table 9. Mauritania: SNIM-Operating Accounts, 1999-2005

(In millions of ouguiya)

\begin{tabular}{lrrrrrrr}
\hline & 1999 & 2000 & 2001 & 2002 & 2003 & 2004 & 2005 \\
& & & & & & & \\
& 41,186 & 54,330 & 57,177 & 44,759 & 48,860 & 69,541 & 109,851 \\
Total revenue & 11,042 & 11,069 & 10,093 & 10,460 & 9,627 & 11,003 & 10,638 \\
$\quad$ Sales (in millions of metric tons) & & & & & & & \\
& 35,772 & 44,976 & 50,472 & 43,750 & 48,163 & 55,032 & 70,832 \\
Total expenses & 16,154 & 20,649 & 22,965 & 22,129 & 24,491 & 27,134 & 36,715 \\
$\quad$ Cost of goods sold & 5,378 & 5,207 & 5,349 & 5,241 & 5,384 & 6,207 & 7,679 \\
$\quad$ Personnel expenses & 2,913 & 2,786 & 77 & 208 & 6,435 & 3,716 & $-1,564$ \\
$\quad$ Financial expenses & 7,934 & 11,413 & 15,739 & 12,287 & 7,566 & 13,215 & 21,008 \\
$\quad$ Depreciation and other provisioning & 436 & 393 & 621 & 119 & 119 & 125 & 134 \\
$\quad$ Taxes & 2,958 & 4,528 & 5,721 & 3,766 & 4,168 & 4,635 & 6,860 \\
$\quad$ Other expenses & 5,414 & 9,354 & 6,705 & 1,009 & 697 & 14,509 & 39,019 \\
Operating profits (+)/losses (-) & & & & & & \\
\hline
\end{tabular}

Source: National Industrial and Mining Company (SNIM).

Table 10. Mauritania: SNIM-Balance Sheet, 2000-05

(In millions of ouguiya; end of period)

\begin{tabular}{|c|c|c|c|c|c|c|}
\hline & 2000 & 2001 & 2002 & 2003 & 2004 & 2005 \\
\hline Assets & 100,905 & 99,936 & 107,311 & 114,462 & 128,406 & 155,450 \\
\hline Cash in banks & 16,791 & 15,110 & 15,964 & 6,432 & 5,747 & 18,062 \\
\hline Receivables & 11,509 & 9,546 & 11,256 & 9,205 & 12,605 & 17,590 \\
\hline Inventories & 10,903 & 14,507 & 11,661 & 15,343 & 16,591 & 23,091 \\
\hline Fixed assets & 56,991 & 59,115 & 68,425 & 83,475 & 91,733 & 95,990 \\
\hline Fixed capital formation expenses & 4,711 & 1,658 & 5 & 7 & 913 & 717 \\
\hline Uncalled capital & $\ldots$ & $\ldots$ & $\ldots$ & $\ldots$ & $\ldots$ & $\ldots$ \\
\hline Other & $\cdots$ & $\cdots$ & $\cdots$ & $\cdots$ & 817 & $\cdots$ \\
\hline Liabilities and equity & 100,905 & 99,936 & 107,311 & 114,461 & 128,405 & 155,450 \\
\hline Short-term debt & 13,775 & 16,433 & 22,546 & 23,326 & 21,633 & 26,132 \\
\hline Long-term debt & 47,667 & 47,315 & 52,405 & 59,426 & 66,816 & 59,489 \\
\hline Equity & 32,309 & 34,521 & 35,571 & 33,098 & 29,150 & 37,324 \\
\hline Losses (-) or profits (+) 1/ & 4,506 & 1,635 & $-3,211$ & $-3,227$ & 8,796 & 29,900 \\
\hline Other & 2,648 & 32 & $\ldots$ & 1,838 & 2,010 & 2,605 \\
\hline
\end{tabular}

Source: National Industrial and Mining Company (SNIM).

1/ After dividends. 
Table 11. Mauritania: Public Utility Rates, 1999-2005

\begin{tabular}{|c|c|c|c|c|c|c|c|}
\hline & 1999 & 2000 & 2001 & 2002 & 2003 & 2004 & 2005 \\
\hline & \multicolumn{7}{|c|}{ (In ouguiya per kilowatt hour) } \\
\hline \multicolumn{8}{|l|}{ Electricity } \\
\hline \multicolumn{8}{|l|}{ Medium voltage (industry) } \\
\hline One hook-up & 20.2 & 23.8 & 23.8 & 23.8 & 23.2 & 23.2 & 26.2 \\
\hline Two hook-ups & 11.9 & 14.9 & 14.9 & 14.9 & 14.9 & 14.9 & 16.1 \\
\hline \multicolumn{8}{|l|}{ Low voltage } \\
\hline Private & 27.6 & 31.7 & 31.7 & 31.7 & 31.7 & 31.7 & 34.7 \\
\hline Public lighting & 31.0 & 35.7 & 35.7 & 37.1 & 37.1 & 37.1 & 41.8 \\
\hline Other & 31.0 & 35.7 & 35.7 & 35.7 & 35.7 & 35.7 & 40.1 \\
\hline & \multicolumn{7}{|c|}{ (In ouguiya per cubic meter) } \\
\hline \multicolumn{8}{|l|}{ Water } \\
\hline \multicolumn{8}{|l|}{ Private } \\
\hline First Tranche & 85.0 & 93.5 & 93.5 & 93.5 & 93.5 & 93.5 & 93.5 \\
\hline Second Tranche & 168.4 & 185.2 & 185.2 & 185.2 & 185.2 & 185.2 & 185.2 \\
\hline Third Tranche & 211.7 & 232.9 & 232.9 & 232.9 & 232.9 & 232.9 & 232.9 \\
\hline Industry and government & 176.4 & 193.6 & 193.6 & 193.6 & 193.6 & 193.6 & 193.6 \\
\hline Public fountains & 77.9 & 85.7 & 85.7 & 85.7 & 85.7 & 85.7 & 85.7 \\
\hline
\end{tabular}

Source: National Electricity Company (SOMELEC).

Table 12. Mauritania: Consumption of Petroleum Products, 1999-2005

(In thousands of metric tons)

\begin{tabular}{|c|c|c|c|c|c|c|c|}
\hline & 1999 & 2000 & 2001 & 2002 & 2003 & 2004 & 2005 \\
\hline Ordinary gasoline & 33.8 & 26.0 & 22.3 & 24.3 & 26.9 & 26.9 & 25.4 \\
\hline Fuel oil & 89.4 & 83.7 & 78.7 & 80.1 & 76.1 & 76.5 & 99.0 \\
\hline Kerosene & 18.1 & 19.9 & 18.7 & 20.1 & 22.8 & 23.1 & 25.1 \\
\hline Lighting oil & 0.8 & 0.3 & 0.2 & 1.3 & 0.8 & 1.3 & 0.9 \\
\hline Gas oil & 213.5 & 224.8 & 246.8 & 277.4 & 289.2 & 317.9 & 308.0 \\
\hline Fishing fleet & 59.7 & 57.7 & 59.7 & 61.2 & 62.8 & 72.2 & 62.1 \\
\hline Transportation & 92.6 & 99.7 & 107.6 & 136.8 & 158.0 & 171.4 & 175.4 \\
\hline SOMELEC & 12.4 & 16.4 & 30.9 & 31.5 & 18.3 & 23.5 & 17.9 \\
\hline SNIM & 48.8 & 51.1 & 48.6 & 47.9 & 50.1 & 50.8 & 52.6 \\
\hline Other & $\ldots$ & $\ldots$ & $\ldots$ & $\cdots$ & $\ldots$ & $\ldots$ & $\ldots$ \\
\hline Butane & 17.4 & 17.4 & 18.1 & 18.5 & 23.1 & 23.6 & 29.2 \\
\hline
\end{tabular}

Source: Ministry of Energy and Petroleum, Directorate of Energy. 
Table 13. Mauritania: Unit Prices of Petroleum Products, 2001-06

(Prices in ouguiya per liter; unless otherwise specified)

\begin{tabular}{|c|c|c|c|c|}
\hline & Regular gasoline & Lighting oil & $\begin{array}{c}\text { Gas oil } \\
\text { (transportation) }\end{array}$ & $\begin{array}{c}\text { Butane } \\
\text { (tank of } 12.5 \mathrm{~kg} \text { ) }\end{array}$ \\
\hline \multicolumn{5}{|l|}{2001} \\
\hline 9-Jan & 173.1 & 108.2 & 119.1 & 1,500 \\
\hline 20-Mar & 173.1 & 108.2 & 119.1 & 1,660 \\
\hline 27-Jun & 171.1 & 107.2 & 118.1 & 1,660 \\
\hline 26-Aug & 167.0 & 101.6 & 113.1 & 1,560 \\
\hline 8-Nov & 152.5 & 90.0 & 105.1 & 1,560 \\
\hline \multicolumn{5}{|l|}{2002} \\
\hline 27-Jan & 149.8 & 83.9 & 100.5 & 1,560 \\
\hline 3-Mar & 137.5 & 83.9 & 97.0 & 1,560 \\
\hline 28-May & 144.6 & 83.9 & 100.2 & 1,560 \\
\hline 1-Sep & 141.5 & 85.1 & 101.4 & 1,560 \\
\hline \multicolumn{5}{|l|}{2003} \\
\hline 16-Jan & 131.9 & 100.2 & 112.8 & 1,560 \\
\hline 3-Apr & 145.7 & 102.4 & 118.8 & 1,560 \\
\hline 7-Aug & 137.7 & 100.9 & 110.8 & 1,560 \\
\hline 19-Oct & 135.7 & 100.9 & 106.8 & 1,560 \\
\hline \multicolumn{5}{|l|}{2004} \\
\hline 3-Feb & 135.7 & 100.9 & 106.8 & 1,560 \\
\hline 6-May & 150.7 & 100.9 & 120.2 & 1,560 \\
\hline 25-Aug & 212.8 & 161.0 & 156.2 & 1,560 \\
\hline \multicolumn{5}{|l|}{2005} \\
\hline $28-F e b$ & 212.8 & 177.0 & 173.1 & 1,560 \\
\hline 6-Jun & 223.7 & 197.2 & 188.8 & 2,100 \\
\hline 30-Dec & 240.7 & 217.3 & 209.3 & 2,100 \\
\hline \multicolumn{5}{|l|}{2006} \\
\hline 28-Feb & 250.2 & 229.6 & 217.7 & 2,100 \\
\hline 2-May & 302.8 & 267.5 & 247.0 & $\ldots$ \\
\hline
\end{tabular}

Source: Ministry of Energy and Petroleum. 


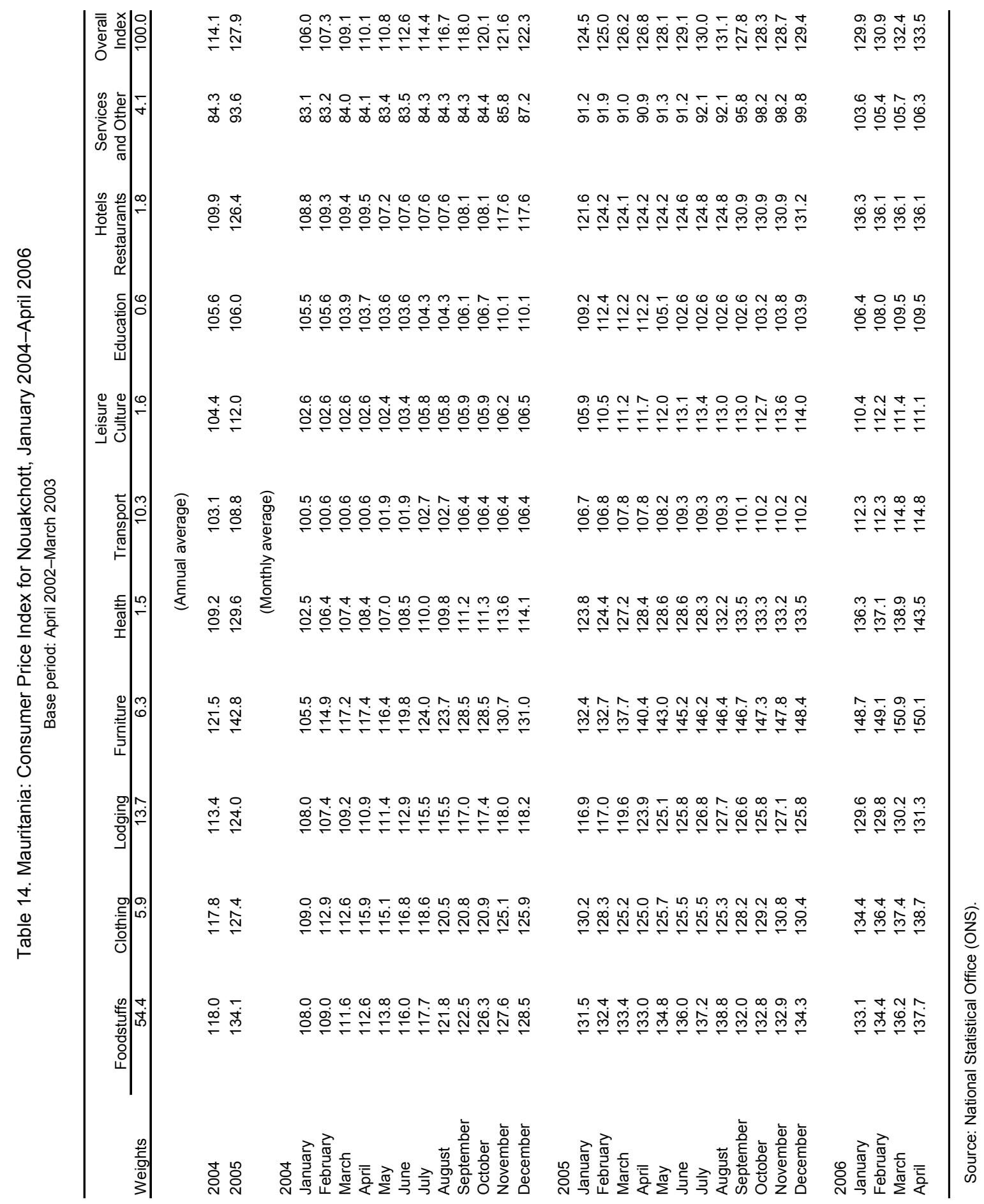


Table 15. Mauritania: Evolution of Minimum Wages and Salaries, 1999-2005

\begin{tabular}{|c|c|c|c|c|c|c|c|}
\hline & 1999 & 2000 & 2001 & 2002 & 2003 & 2004 & 2005 \\
\hline & \multicolumn{7}{|c|}{ (In ouguiya) } \\
\hline \multicolumn{8}{|c|}{ Private sector (hourly wages) } \\
\hline SMIG 1/ & 64.5 & 66.5 & 69.0 & 69.0 & 69.0 & 82.7 & 128.8 \\
\hline SMAG 2/ & 64.5 & 66.5 & 69.0 & 69.0 & 69.0 & 82.7 & 128.8 \\
\hline \multicolumn{8}{|c|}{ Public sector (civil servants' monthly salary) } \\
\hline Category A1 & 20,844 & 21,791 & 22,976 & 22,976 & 22,976 & 28,149 & 36,149 \\
\hline Category A2 & 17,852 & 18,550 & 19,423 & 19,423 & 19,423 & 23,601 & 31,601 \\
\hline Category B & 15,944 & 16,543 & 17,291 & 17,291 & 17,291 & 21,351 & 29,351 \\
\hline Category C & 11,176 & 11,525 & 11,961 & 11,961 & 11,961 & 14,330 & 22,330 \\
\hline Category D & 9,876 & 10,225 & 10,661 & 10,661 & 10,661 & 13,030 & 21,030 \\
\hline \multicolumn{8}{|l|}{ Category E (teachers) } \\
\hline Assistant teachers & 16,176 & 16,525 & 16,961 & 16,961 & 16,961 & 19,330 & 27,330 \\
\hline Teachers & 24,852 & 25,550 & 26,423 & 26,423 & 26,423 & 30,601 & 38,601 \\
\hline Assistant professors & 30,352 & 31,050 & 31,923 & 31,923 & 31,923 & 36,101 & 44,101 \\
\hline Professors & 35,844 & 36,791 & 37,976 & 37,976 & 37,976 & 43,149 & 51,149 \\
\hline & \multicolumn{7}{|c|}{ (In U.S. dollars) } \\
\hline \multicolumn{8}{|c|}{ Private sector (hourly wages) } \\
\hline SMIG 1/ & 0.31 & 0.28 & 0.27 & 0.25 & 0.26 & 0.31 & 0.49 \\
\hline SMAG 2/ & 0.31 & 0.28 & 0.27 & 0.25 & 0.26 & 0.31 & 0.49 \\
\hline \multicolumn{8}{|c|}{ Public sector (civil servants' monthly salary) } \\
\hline Category A1 & 99 & 91 & 90 & 85 & 87 & 106 & 136 \\
\hline Category A2 & 85 & 78 & 76 & 71 & 74 & 89 & 119 \\
\hline Category B & 76 & 69 & 68 & 64 & 66 & 80 & 111 \\
\hline Category C & 53 & 48 & 47 & 44 & 45 & 54 & 84 \\
\hline Category D & 47 & 43 & 42 & 39 & 41 & 49 & 79 \\
\hline \multicolumn{8}{|l|}{ Category E (teachers) } \\
\hline Assistant teachers & 77 & 69 & 66 & 62 & 64 & 73 & 103 \\
\hline Teachers & 119 & 107 & 103 & 97 & 100 & 115 & 145 \\
\hline Assistant professors & 145 & 130 & 125 & 117 & 121 & 136 & 166 \\
\hline Professors & 171 & 154 & 149 & 140 & 144 & 163 & 193 \\
\hline
\end{tabular}

Source: Ministry of Finance, Directorate of Budget.

1/ Guaranteed minimum industrial wage.

2/ Guaranteed minimum agricultural wage. 


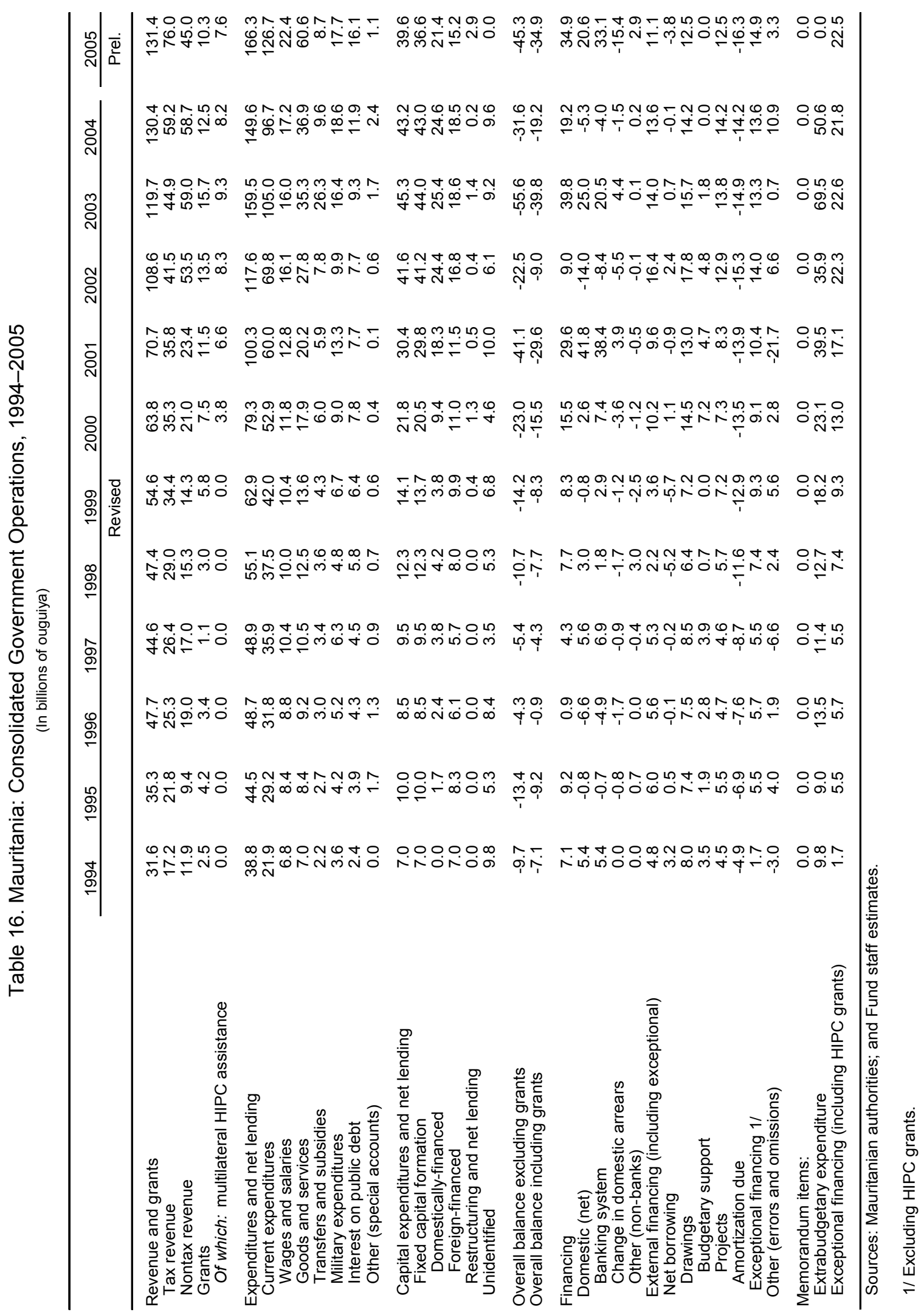




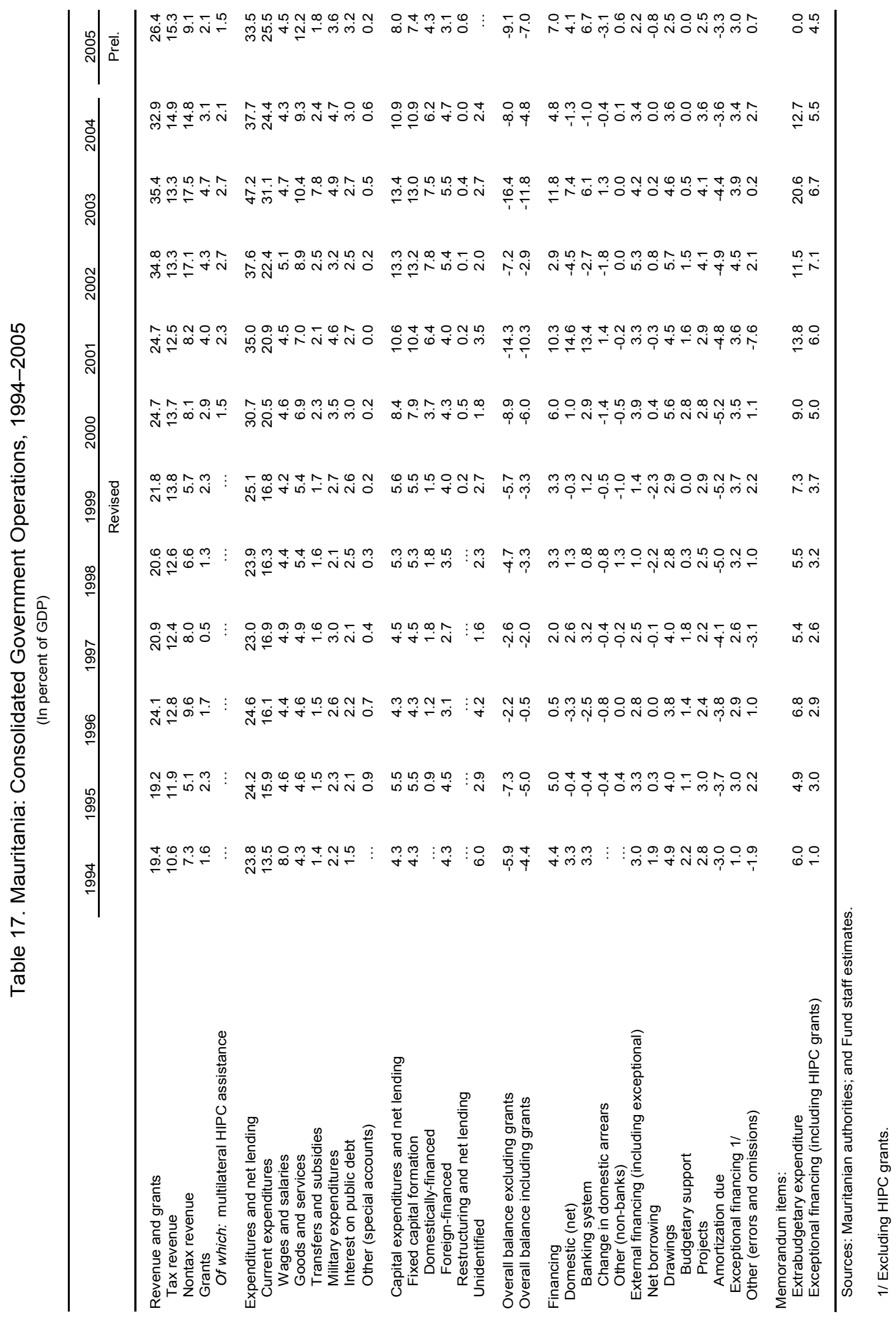




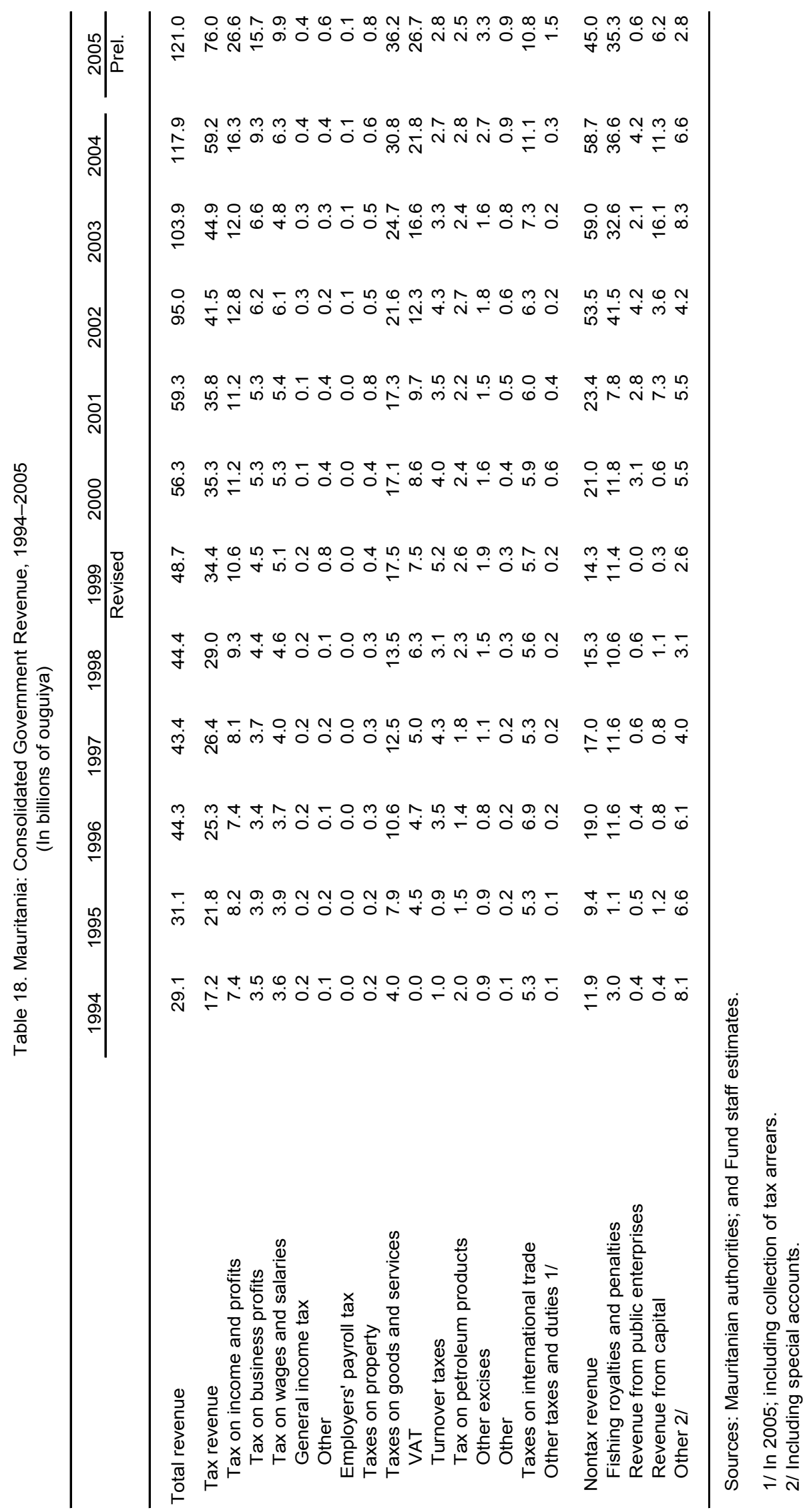




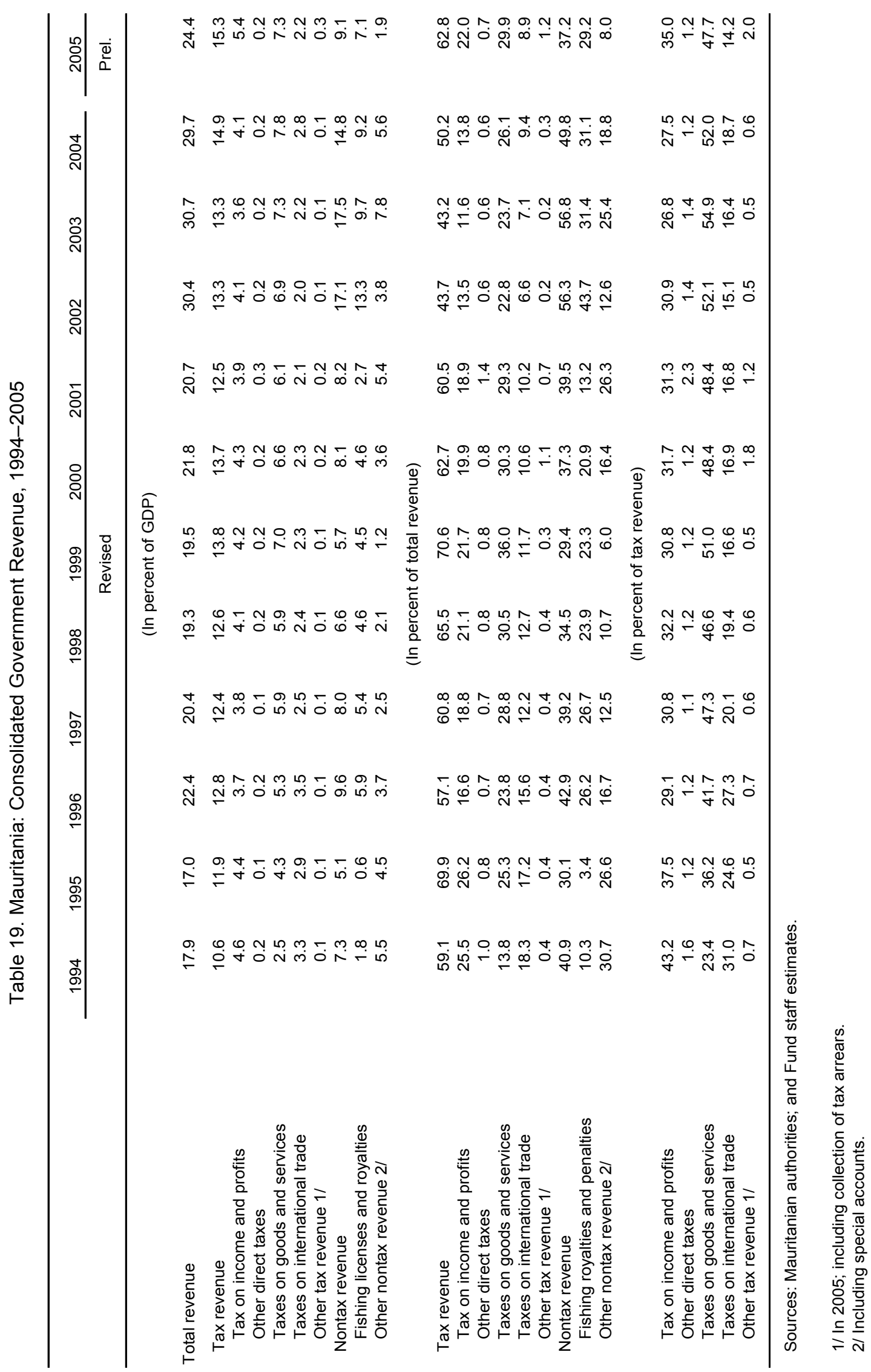




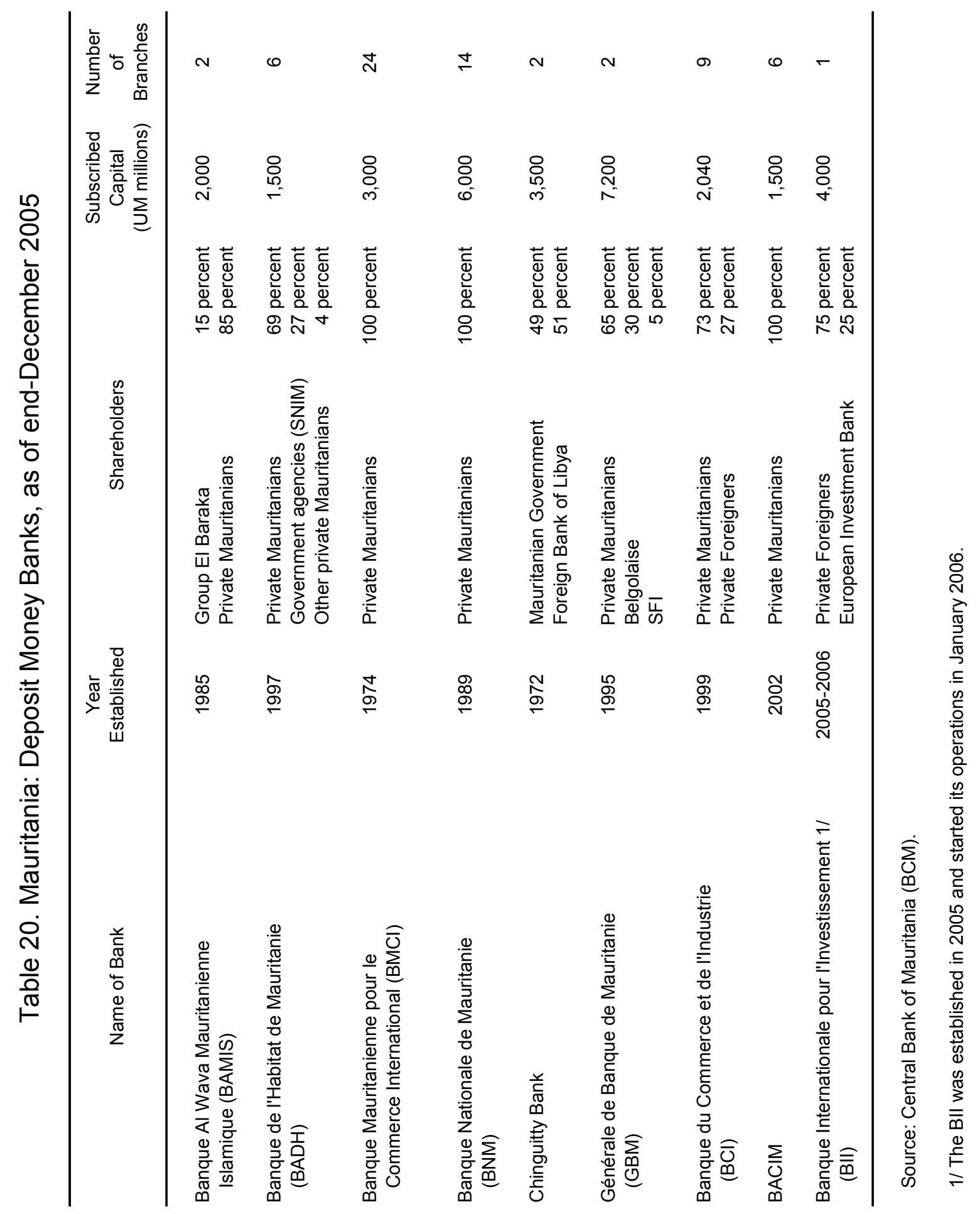




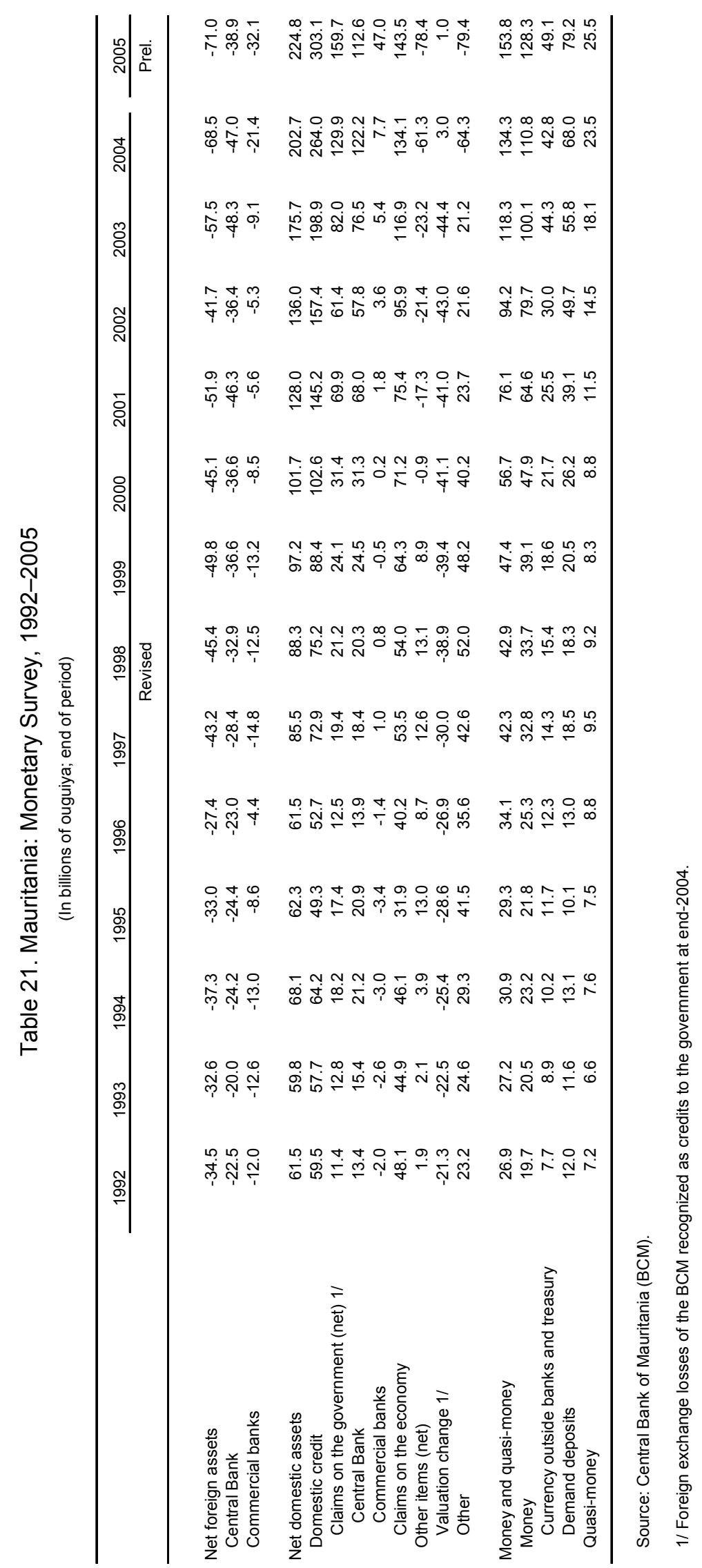




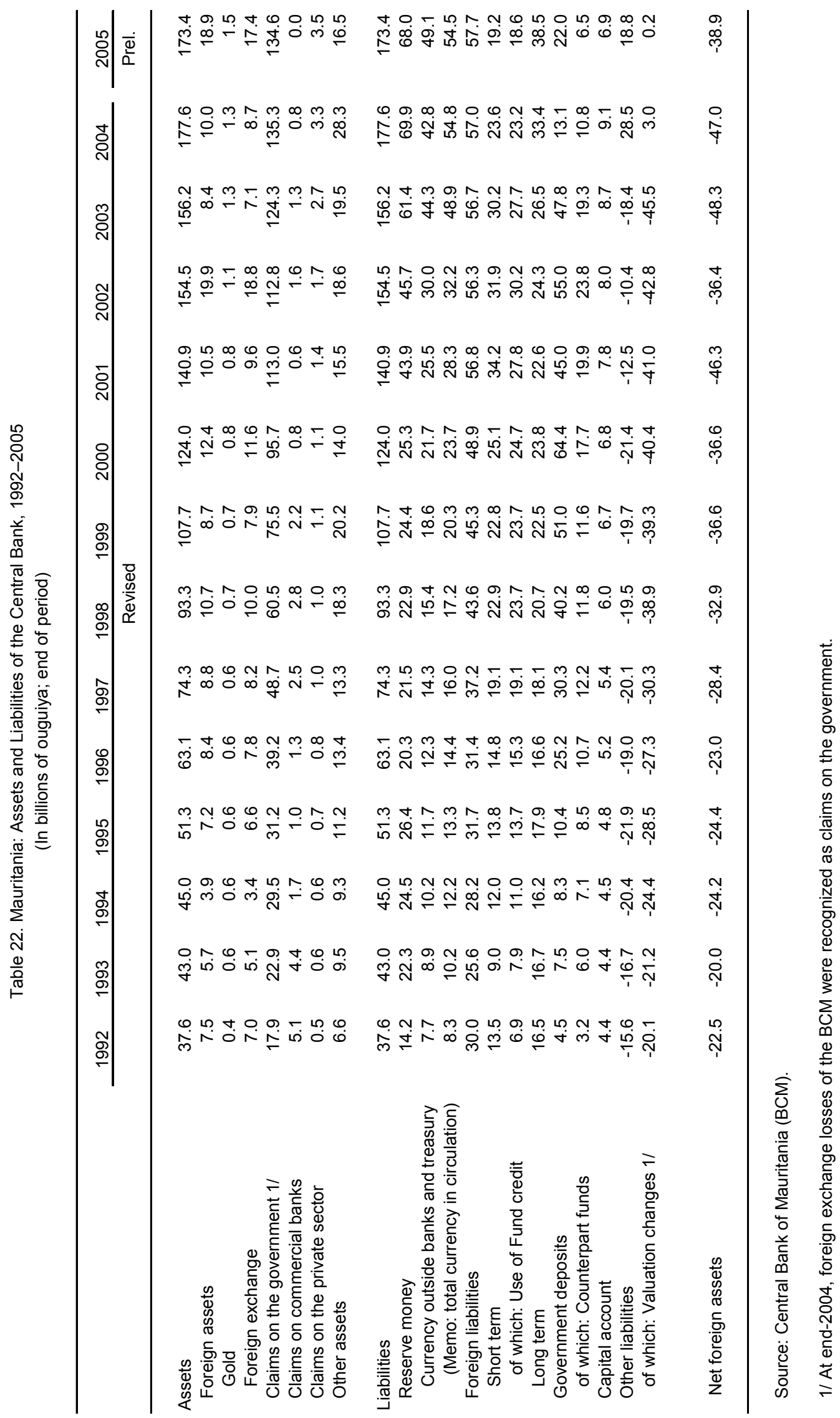




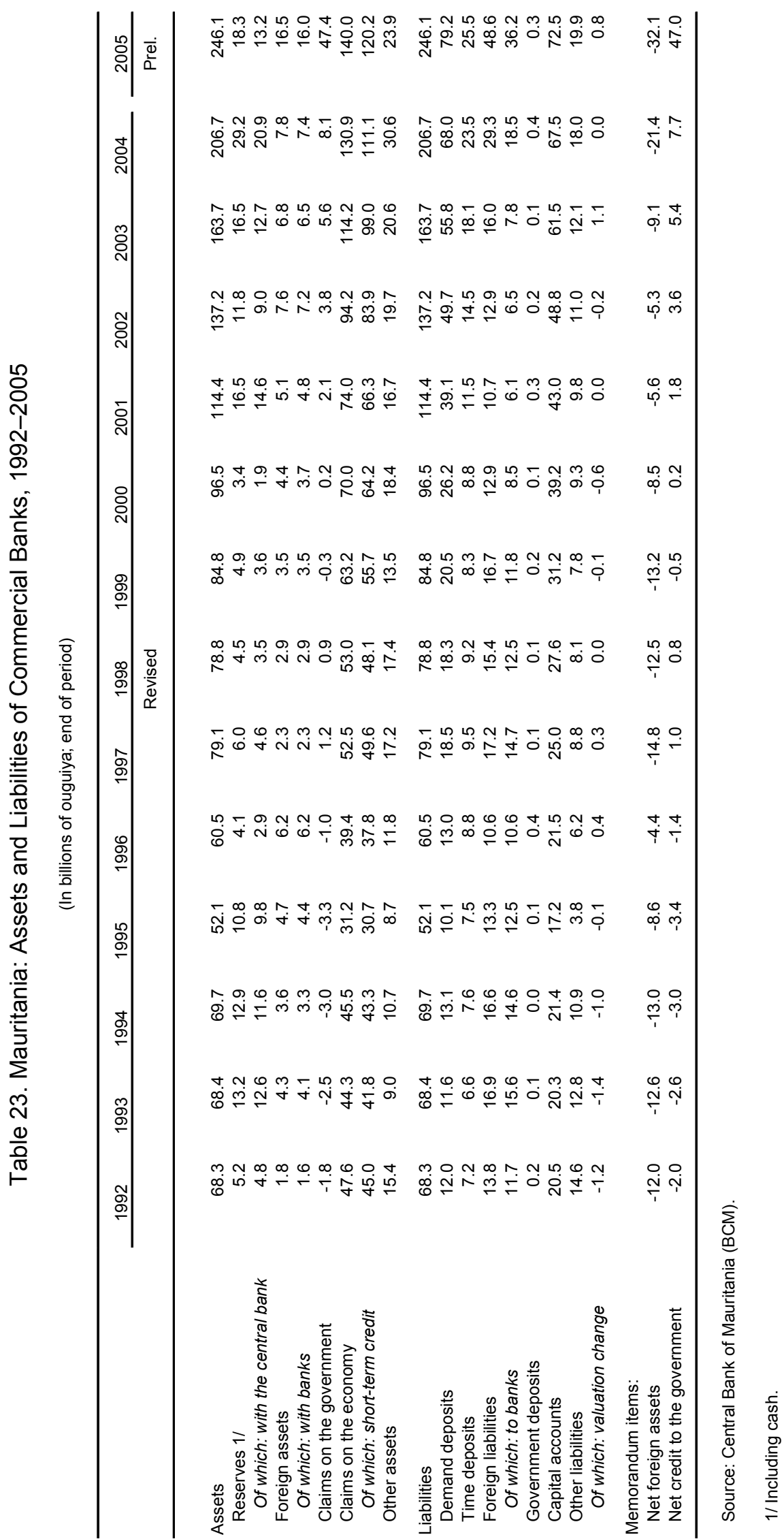




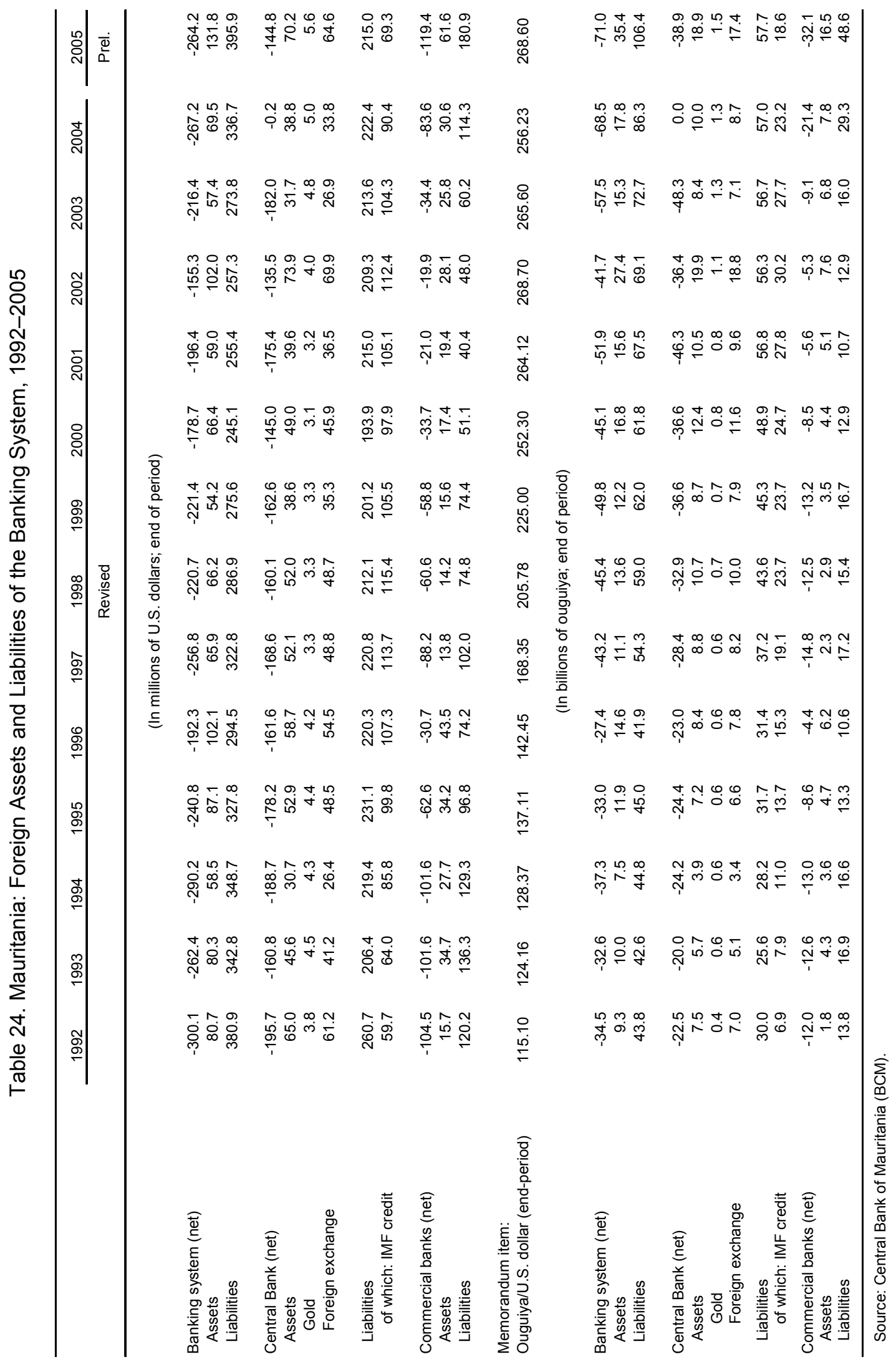




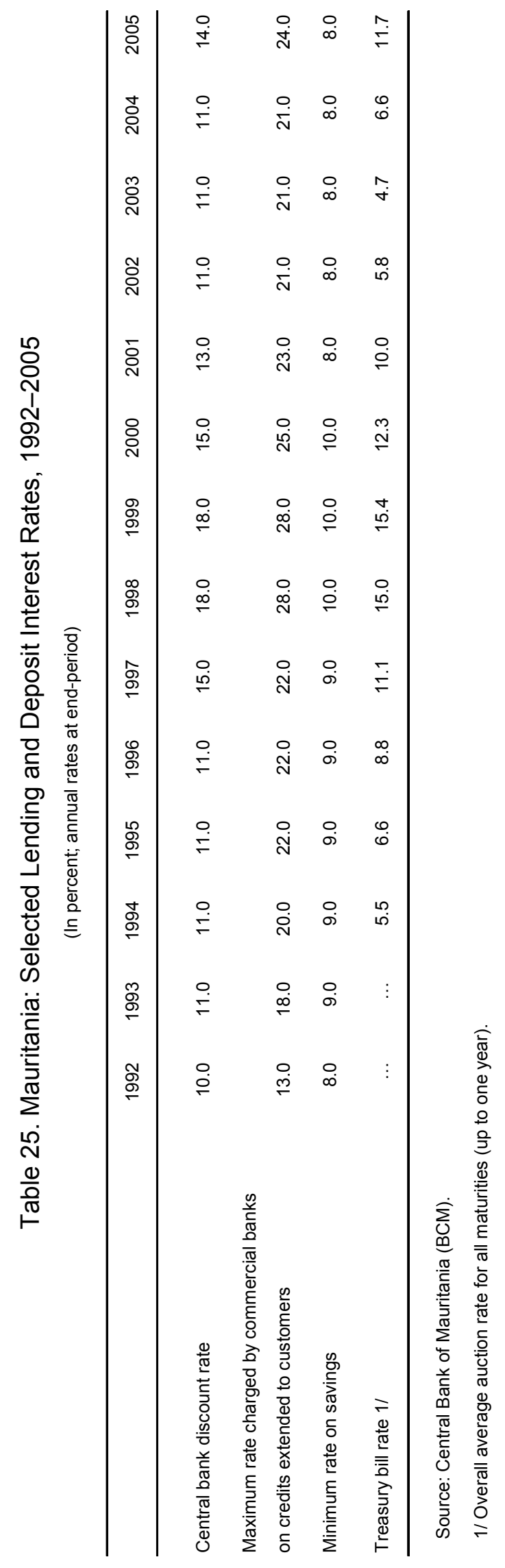


Table 26. Mauritania: Distribution of Bank Credit by Maturity and Economic Activity, 1992-2005

\begin{tabular}{|c|c|c|c|c|c|c|c|c|c|c|c|c|c|c|}
\hline & 1992 & 1993 & 1994 & 1995 & 1996 & 1997 & 1998 & 1999 & 2000 & 2001 & 2002 & 2003 & 2004 & 2005 \\
\hline & \multicolumn{13}{|c|}{ Revised } & Prel. \\
\hline \multicolumn{15}{|c|}{ (In billions of ouguiya; end of period) } \\
\hline Total & 45.2 & 40.6 & 41.3 & 27.3 & 33.5 & 46.3 & 46.5 & 52.0 & 59.8 & 64.9 & 84.9 & 106.3 & 119.3 & 125.5 \\
\hline Short term & 43.2 & 38.8 & 39.8 & 27.1 & 32.6 & 44.3 & 42.8 & 45.8 & 55.5 & 59.2 & 76.8 & 93.1 & 101.7 & 108.2 \\
\hline Agriculture and livestock & 0.6 & 0.6 & 0.6 & 0.3 & 0.4 & 0.1 & 0.1 & 1.1 & 0.2 & 0.2 & 1.4 & 1.1 & 1.3 & 0.9 \\
\hline Fishing & 13.5 & 13.0 & 12.8 & 9.0 & 11.6 & 11.8 & 12.3 & 11.9 & 13.2 & 11.4 & 13.8 & 13.9 & 15.3 & 16.0 \\
\hline Mining & 0.1 & 0.2 & 0.2 & 0.3 & 0.0 & 0.0 & 0.0 & 0.0 & 0.0 & 0.0 & 0.0 & 0.0 & 0.0 & 0.0 \\
\hline Manufacturing & 2.6 & 1.8 & 1.5 & 0.7 & 2.3 & 1.9 & 1.8 & 1.9 & 1.8 & 1.4 & 2.2 & 3.2 & 3.0 & 3.2 \\
\hline Construction & 1.2 & 1.1 & 1.3 & 0.7 & 1.1 & 1.1 & 1.4 & 1.8 & 2.9 & 4.0 & 5.1 & 10.5 & 14.3 & 15.7 \\
\hline Transport & 1.0 & 1.1 & 1.0 & 0.7 & 0.9 & 1.0 & 0.9 & 0.8 & 1.0 & 1.8 & 1.7 & 2.5 & 1.9 & 4.0 \\
\hline Trade & 12.0 & 11.7 & 9.3 & 6.3 & 8.9 & 19.6 & 18.5 & 18.2 & 23.0 & 25.3 & 29.9 & 29.7 & 30.9 & 27.2 \\
\hline Services & 3.2 & 2.0 & 2.5 & 1.8 & 3.1 & 3.6 & 2.7 & 2.8 & 4.9 & 6.2 & 12.0 & 17.0 & 15.8 & 18.6 \\
\hline Other & 8.9 & 7.4 & 10.7 & 7.2 & 4.5 & 5.2 & 5.0 & 7.2 & 8.4 & 9.0 & 10.6 & 15.2 & 19.1 & 22.4 \\
\hline Medium term & 2.0 & 1.8 & 1.5 & 0.3 & 0.9 & 2.0 & 3.8 & 6.2 & 4.3 & 5.6 & 8.1 & 13.2 & 17.6 & 17.3 \\
\hline Agriculture and livestock & 0.2 & 0.2 & 0.2 & 0.0 & 0.0 & 0.0 & 0.0 & 0.0 & 0.0 & 0.0 & 0.0 & 0.0 & 0.0 & 0.0 \\
\hline Fishing & 0.9 & 0.6 & 0.7 & 0.1 & 0.4 & 0.7 & 1.0 & 2.1 & 1.0 & 1.3 & 1.4 & 2.6 & 3.6 & 3.8 \\
\hline Mining & 0.0 & 0.0 & 0.0 & 0.0 & 0.0 & 0.0 & 0.0 & 0.0 & 0.0 & 0.0 & 0.0 & 0.0 & 0.0 & 0.0 \\
\hline Manufacturing & 0.3 & 0.3 & 0.2 & 0.0 & 0.0 & 0.2 & 0.4 & 0.5 & 0.4 & 0.5 & 1.0 & 1.3 & 1.3 & 1.3 \\
\hline Construction & 0.0 & 0.0 & 0.0 & 0.0 & 0.0 & 0.0 & 0.0 & 0.2 & 0.2 & 0.3 & 0.3 & 0.3 & 0.1 & 0.2 \\
\hline Transport & 0.0 & 0.0 & 0.0 & 0.0 & 0.0 & 0.0 & 0.0 & 0.0 & 0.0 & 0.0 & 0.0 & 0.2 & 0.8 & 1.9 \\
\hline Trade & 0.0 & 0.0 & 0.0 & 0.0 & 0.2 & 0.4 & 0.6 & 2.2 & 1.4 & 1.6 & 2.5 & 2.8 & 3.0 & 2.6 \\
\hline Services & 0.3 & 0.3 & 0.3 & 0.0 & 0.1 & 0.3 & 1.0 & 1.0 & 1.1 & 1.8 & 2.7 & 5.6 & 8.5 & 7.1 \\
\hline Other & 0.3 & 0.3 & 0.1 & 0.1 & 0.2 & 0.3 & 0.8 & 0.3 & 0.2 & 0.2 & 0.2 & 0.4 & 0.4 & 0.4 \\
\hline \multicolumn{15}{|c|}{ (In percent of total bank credit) } \\
\hline Agriculture and livestock & 1.9 & 2.0 & 1.9 & 1.4 & 1.1 & 0.3 & 0.3 & 2.0 & 0.4 & 0.3 & 1.7 & 1.1 & 1.1 & 0.7 \\
\hline Fishing & 31.9 & 33.6 & 32.7 & 33.4 & 35.8 & 27.0 & 28.6 & 26.9 & 23.7 & 19.5 & 17.9 & 15.5 & 15.9 & 15.8 \\
\hline Mining & 0.3 & 0.4 & 0.5 & 0.9 & 0.0 & 0.0 & 0.0 & 0.0 & 0.0 & 0.0 & 0.0 & 0.0 & 0.0 & 0.0 \\
\hline Manufacturing & 6.5 & 5.0 & 4.0 & 2.6 & 6.9 & 4.6 & 4.7 & 4.7 & 3.7 & 3.0 & 3.8 & 4.2 & 3.6 & 3.6 \\
\hline Construction & 2.7 & 2.8 & 3.2 & 2.4 & 3.1 & 2.3 & 3.2 & 3.8 & 5.2 & 6.6 & 6.5 & 10.2 & 12.1 & 12.6 \\
\hline Transport & 2.1 & 2.7 & 2.5 & 2.7 & 2.6 & 2.1 & 1.9 & 1.6 & 1.7 & 2.8 & 2.1 & 2.6 & 2.3 & 4.7 \\
\hline Trade & 26.6 & 28.9 & 22.5 & 23.2 & 27.0 & 43.4 & 41.0 & 39.2 & 40.8 & 41.4 & 38.2 & 30.6 & 28.4 & 23.8 \\
\hline Services & 7.7 & 5.7 & 6.7 & 6.6 & 9.3 & 8.5 & 8.0 & 7.4 & 10.1 & 12.3 & 17.3 & 21.2 & 20.3 & 20.5 \\
\hline Other & 20.3 & 19.0 & 25.9 & 26.7 & 14.2 & 11.8 & 12.4 & 14.4 & 14.5 & 14.1 & 12.6 & 14.7 & 16.3 & 18.1 \\
\hline Total & 100.0 & 100.0 & 100.0 & 100.0 & 100.0 & 100.0 & 100.0 & 100.0 & 100.0 & 100.0 & 100.0 & 100.0 & 100.0 & 100.0 \\
\hline Short term & 95.5 & 95.6 & 96.4 & 99.0 & 97.3 & 95.8 & 91.9 & 88.1 & 92.8 & 91.3 & 90.4 & 87.6 & 85.2 & 86.2 \\
\hline Medium term & 4.5 & 4.4 & 3.6 & 1.0 & 2.7 & 4.2 & 8.1 & 11.9 & 7.2 & 8.7 & 9.6 & 12.4 & 14.8 & 13.8 \\
\hline
\end{tabular}

Source: Central Bank of Mauritania (BCM). 


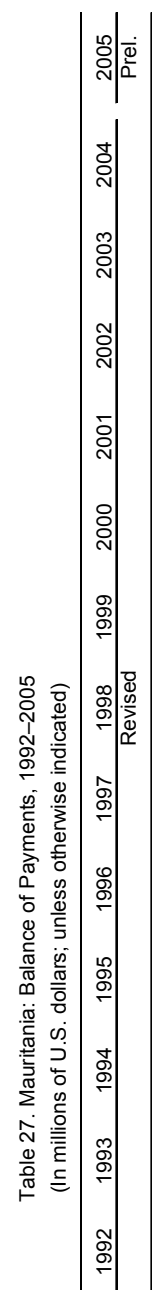

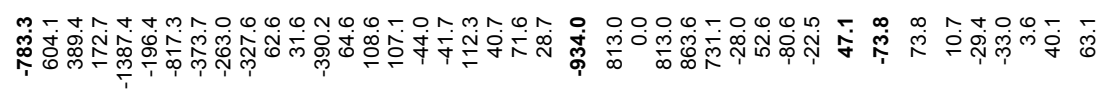

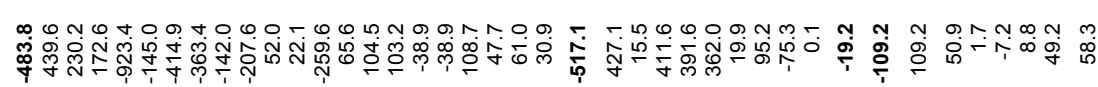

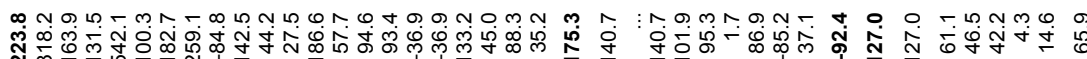
สู่

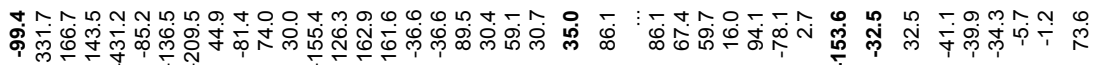

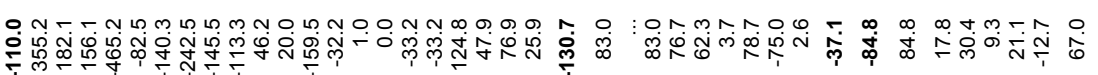

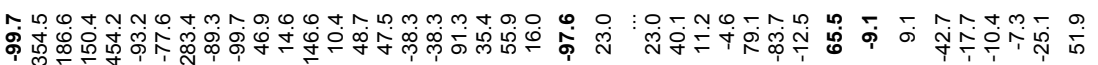

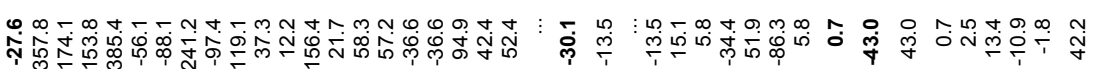
0人

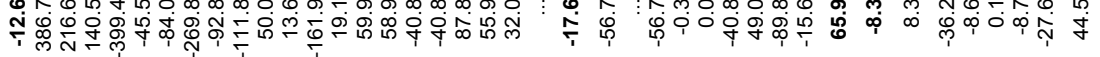
ช்

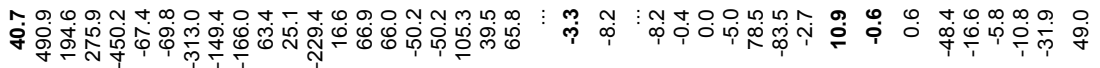

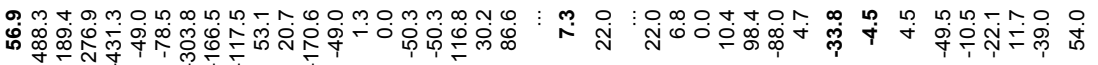
ล் m

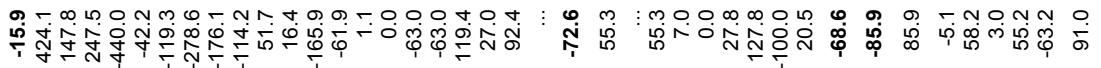
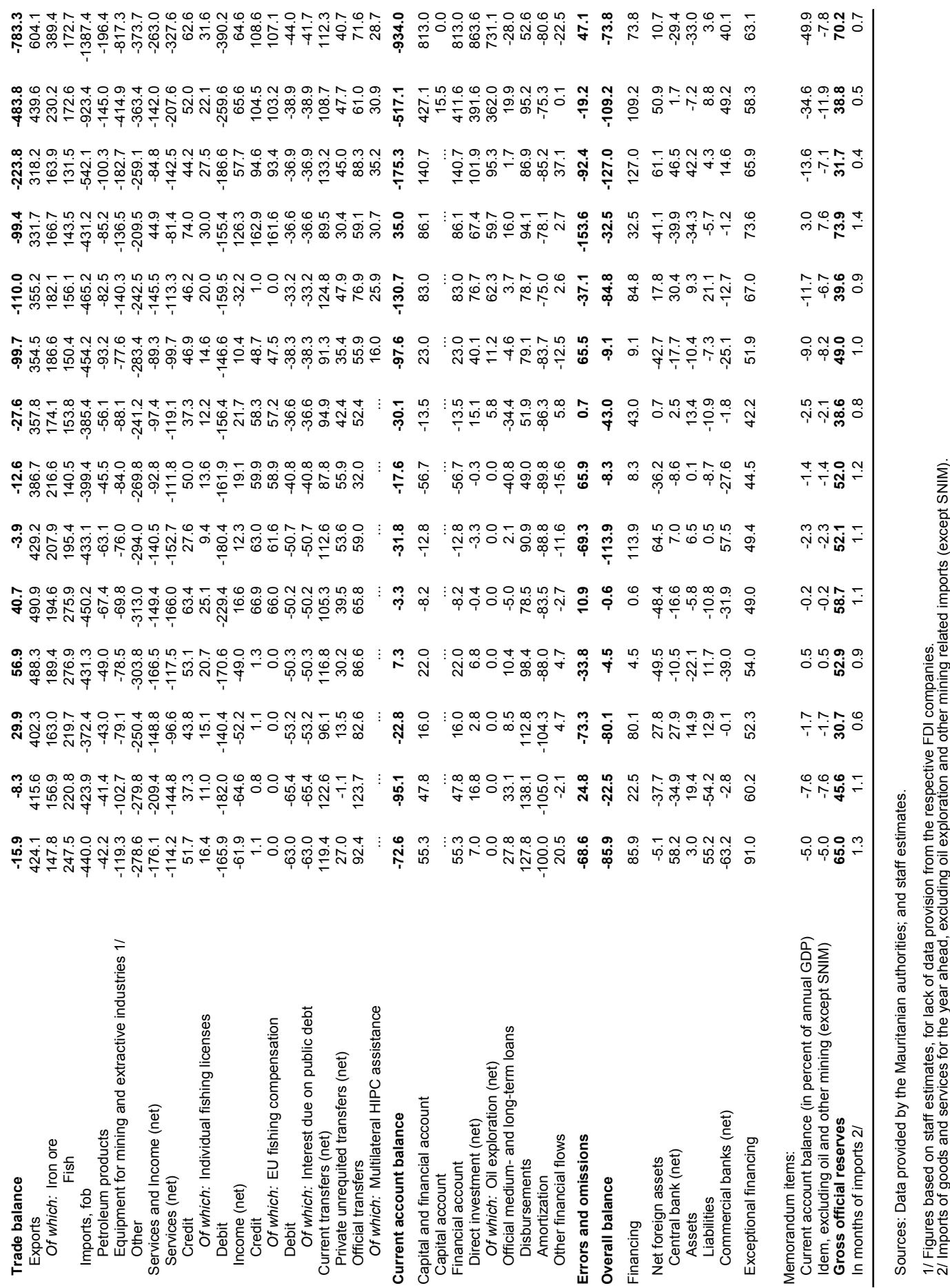


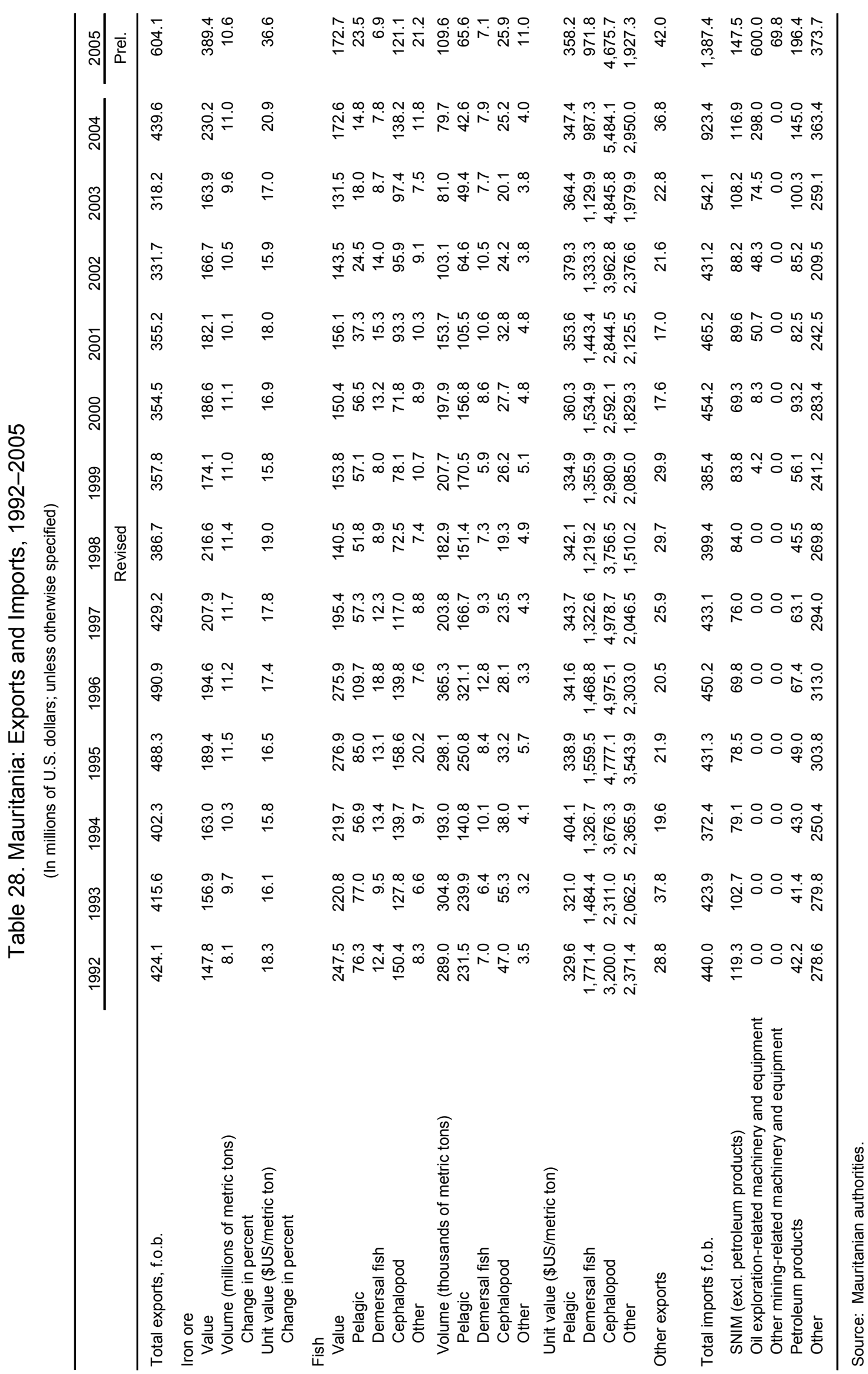




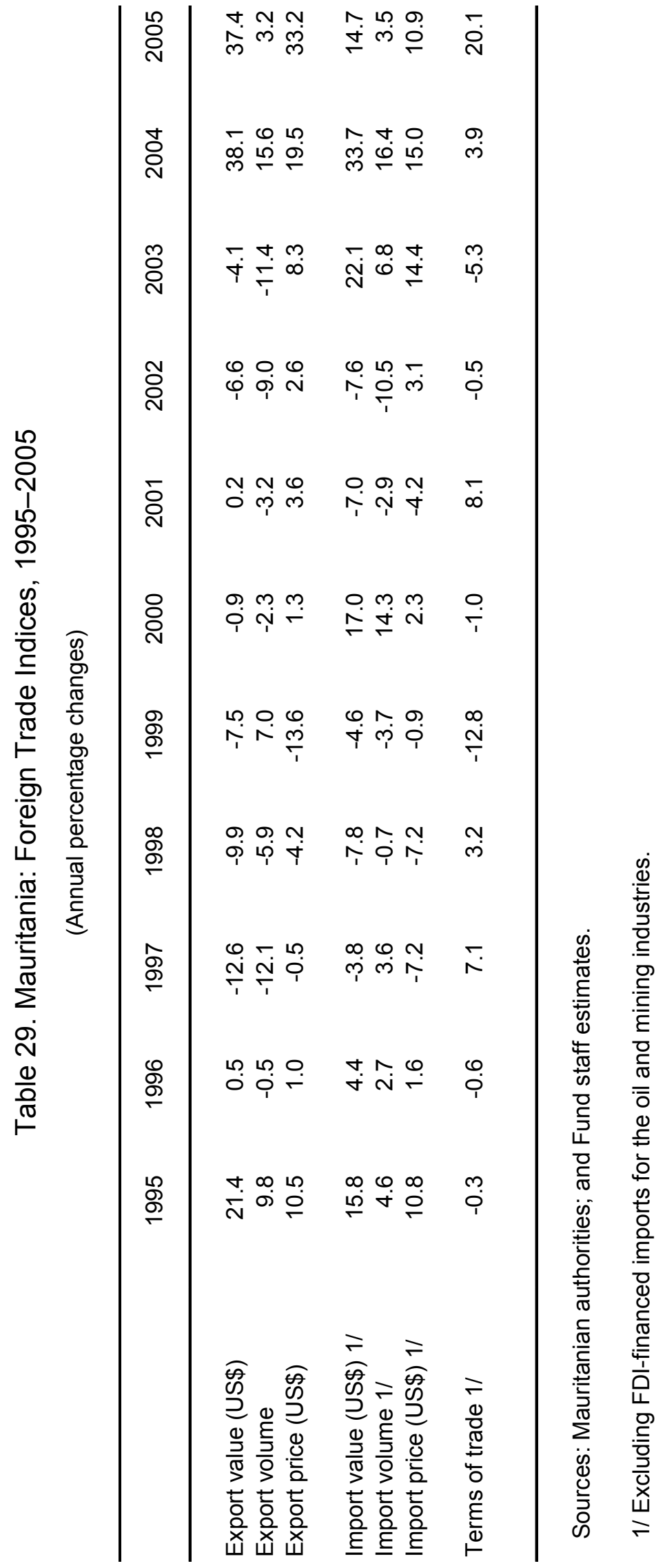




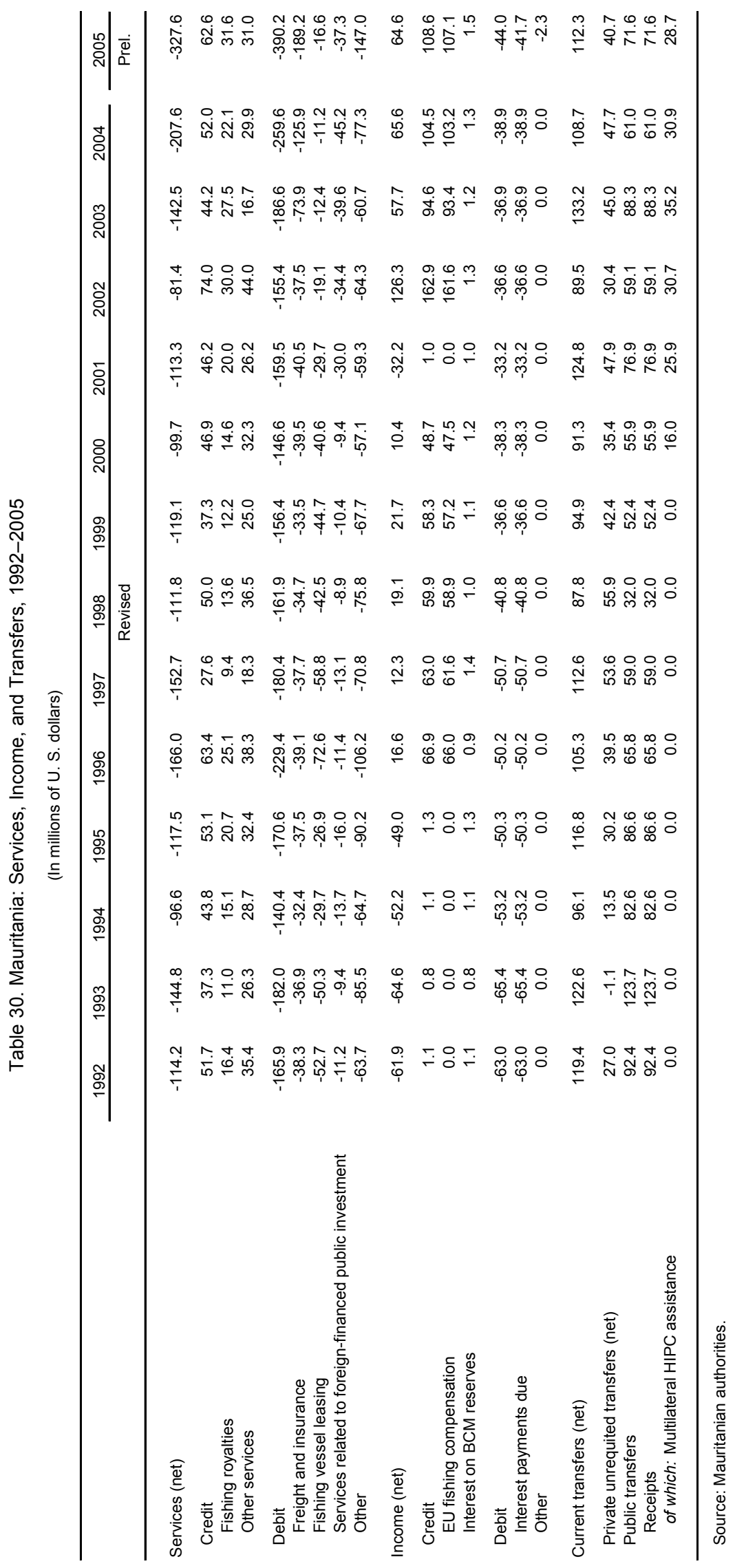




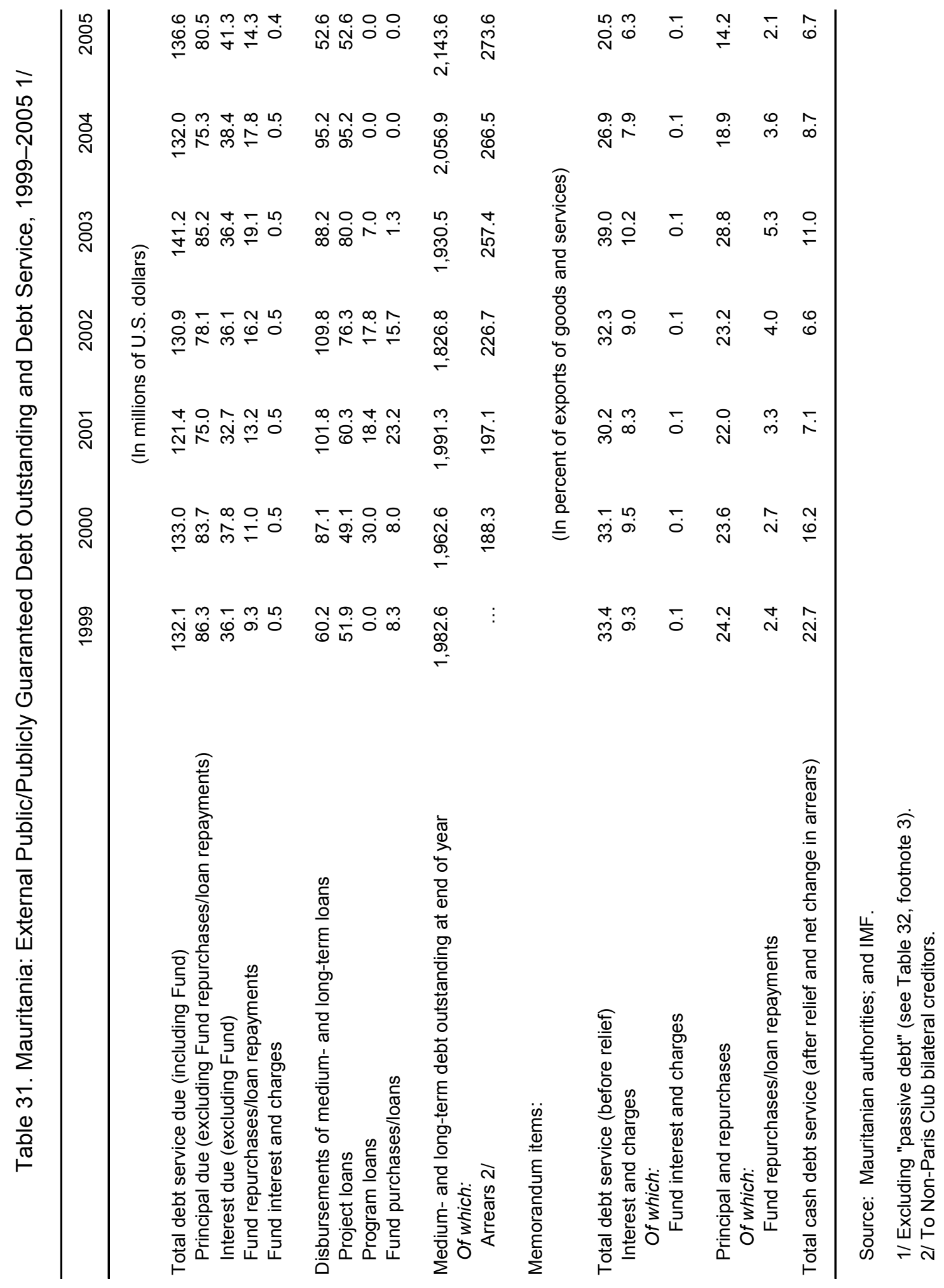


Table 32. Mauritania: Medium- and Long-Term External Public/Publicly Guaranteed Debt, 1999-2005 (In millions of U.S. dollars; end of period)

\begin{tabular}{|c|c|c|c|c|c|c|c|}
\hline & 1999 & 2000 & 2001 & 2002 & 2003 & 2004 & 2005 \\
\hline Total $1 /$ & $1,982.6$ & $1,962.6$ & $1,991.3$ & $1,826.8$ & $1,930.5$ & $2,056.9$ & $2,143.6$ \\
\hline Bilateral loans $1 /$ & 971.7 & 915.9 & 826.4 & 551.1 & 499.9 & 509.4 & 661.4 \\
\hline Algeria & 70.3 & 72.2 & 71.0 & 72.0 & 72.0 & 72.6 & 93.4 \\
\hline Austria & 86.5 & 68.1 & 58.6 & 0.0 & 0.0 & 0.0 & 0.0 \\
\hline China, P.R. & 76.9 & 78.7 & 48.9 & 45.9 & 49.3 & 49.7 & 52.4 \\
\hline France & 137.7 & 116.5 & 78.4 & 35.6 & 19.9 & 18.4 & 13.8 \\
\hline Germany & 5.1 & 3.6 & 5.0 & 2.5 & 2.5 & 5.1 & 4.5 \\
\hline Japan & 72.6 & 73.5 & 64.6 & 0.0 & 0.0 & 0.0 & 0.0 \\
\hline Netherlands & 34.5 & 64.5 & 20.0 & 0.0 & 0.0 & 0.0 & 0.0 \\
\hline Saudi Arabia & 130.7 & 143.2 & 141.5 & 141.7 & 142.0 & 143.2 & 141.9 \\
\hline Spain & 39.1 & 41.3 & 44.0 & 32.9 & 20.0 & 25.3 & 42.4 \\
\hline United Arab Emirates & 10.6 & 12.1 & 10.9 & 11.7 & 8.5 & 8.6 & 12.5 \\
\hline Others & 307.8 & 242.2 & 283.5 & 208.7 & 185.7 & 186.4 & 300.6 \\
\hline Multilateral loans $1 /$ & $1,010.9$ & $1,046.6$ & $1,164.9$ & $1,275.7$ & $1,430.6$ & $1,547.5$ & $1,482.2$ \\
\hline African Development Bank & 49.6 & 42.1 & 42.0 & 44.6 & 52.6 & 61.7 & 53.7 \\
\hline African Development Fund & 159.8 & 192.8 & 186.1 & 196.9 & 224.1 & 259.2 & 242.9 \\
\hline AFESD 2/ & 122.6 & 138.1 & 160.0 & 187.5 & 222.9 & 248.9 & 255.9 \\
\hline Arab Monetary Fund & 20.3 & 25.5 & 20.1 & 17.7 & 13.9 & 9.4 & 19.7 \\
\hline European Investment Bank & 67.8 & 48.1 & 61.1 & 45.1 & 28.5 & 22.2 & 14.4 \\
\hline IBRD & 1.9 & 0.0 & 0.0 & 0.0 & 0.0 & 0.0 & 0.0 \\
\hline IDA & 395.0 & 405.2 & 469.0 & 545.0 & 636.9 & 696.9 & 674.2 \\
\hline IMF & 106.6 & 97.5 & 105.1 & 111.1 & 103.0 & 89.6 & 69.4 \\
\hline Islamic Development Bank & 38.9 & 39.7 & 38.0 & 51.2 & 68.1 & 76.6 & 76.9 \\
\hline OPEC Special Fund & 16.1 & 13.7 & 16.0 & 25.1 & 25.1 & 25.2 & 25.6 \\
\hline Others & 32.5 & 43.8 & 67.6 & 51.5 & 55.5 & 57.7 & 49.4 \\
\hline \multicolumn{8}{|l|}{ Memorandum items: } \\
\hline Arrears & $\ldots$ & 188.3 & 197.1 & 226.7 & 257.4 & 266.5 & 273.6 \\
\hline Passive debt $3 /$ & 452.3 & 508.7 & 572.5 & 644.6 & 726.1 & 818.1 & 922.1 \\
\hline Kuwait & 434.3 & 490.7 & 554.5 & 626.6 & 708.1 & 800.1 & 904.1 \\
\hline Libya & 10.8 & 10.8 & 10.8 & 10.8 & 10.8 & 10.8 & 10.8 \\
\hline OAPEC 4/ & 7.2 & 7.2 & 7.2 & 7.2 & 7.2 & 7.2 & 7.2 \\
\hline
\end{tabular}

Sources: Mauritanian authorities; and IMF.

$1 /$ Including all arrears except for passive debt.

2/ Arab Fund for Economic and Social Development.

3/ Debt (in arrears) that was excluded from HIPC relief calculations at the decision point and whose treatment remains to be clarified.

4/ Organization of Arab Petroleum Exporting Countries. 
Table 33. Mauritania: Average Terms of Contracted Public External Debt, 1999-2005 1/

(In percent; unless otherwise specified)

\begin{tabular}{|c|c|c|c|c|c|c|c|}
\hline & 1999 & 2000 & 2001 & 2002 & 2003 & 2004 & 2005 \\
\hline \multicolumn{8}{|l|}{ Total loans } \\
\hline Interest rate & 1.4 & 1.6 & 1.3 & 1.6 & 0.8 & 2.5 & 1.0 \\
\hline Maturity (years) & 32.1 & 28.8 & 31.8 & 20.1 & 24.5 & 25.5 & 20.9 \\
\hline Grace period (years) & 8.4 & 8.1 & 7.8 & 7.1 & 12.2 & 6.8 & 4.4 \\
\hline Grant element & n.a. & n.a. & n.a. & n.a. & n.a. & n.a. & n.a. \\
\hline \multicolumn{8}{|l|}{ Multilateral loans } \\
\hline Interest rate & 1.3 & 1.6 & 1.2 & 1.6 & 0.9 & 2.6 & 1.3 \\
\hline Maturity (years) & 32.5 & 28.8 & 34.6 & 20.1 & 26.6 & 26.9 & 25.0 \\
\hline Grace period (years) & 8.4 & 8.1 & 8.2 & 7.1 & 9.0 & 7.5 & 5.8 \\
\hline Grant element & n.a. & n.a. & n.a. & n.a. & n.a. & n.a. & n.a. \\
\hline \multicolumn{8}{|l|}{ Bilateral loans } \\
\hline Interest rate & 2.2 & 2.0 & 1.5 & 1.5 & 0.8 & 2.1 & 0.8 \\
\hline Maturity (years) & 23.5 & 25.0 & 27.0 & 27.0 & 23.5 & 21.7 & 17.0 \\
\hline Grace period (years) & 7.6 & 7.4 & 7.3 & 7.3 & 15.0 & 5.0 & 3.1 \\
\hline Grant element & n.a. & n.a. & n.a. & n.a. & n.a. & n.a. & n.a. \\
\hline
\end{tabular}

Source: Mauritanian authorities.

1/ Based on new commitments. 